\title{
Mutations in the Predicted Active Site of Xanthomonas oryzae pv. oryzae XopQ Differentially Affect Virulence, Suppression of Host Innate Immunity, and Induction of the HR in a Nonhost Plant
}

\author{
Mahesh Kumar Gupta, Rajkanwar Nathawat, Dipanwita Sinha, Asfarul S. Haque, \\ Rajan Sankaranarayanan, and Ramesh V. Sonti \\ CSIR-Centre for Cellular and Molecular Biology, Uppal Road, Hyderabad-500 007, Andhra Pradesh, India
}

Submitted 29 September 2014. Accepted 22 October 2014.

\begin{abstract}
Xanthomonas oryzae pv. oryzae, the bacterial blight pathogen of rice, secretes a number of effectors through a type 3 secretion system. One of these effectors, called XopQ, is required for virulence and suppression of rice innate immune responses induced by the plant cell-wall-degrading enzyme lipase/esterase A (LipA). Bioinformatic analysis suggested that XopQ is homologous to inosine-uridine nucleoside hydrolases (NH). A structural model of XopQ with the protozoan Crithidia fasciculata purine NH suggested that D116 and Y279 are potential active site residues. $X$. oryzae pv. oryzae xop $Q$ mutants (xop $Q^{-/ p H M 1:: x o p} Q^{D 116 A}$ and $x o p Q^{-} / \mathrm{pHM1}: \operatorname{xop} Q^{Y 279 A}$ ) show reduced virulence on rice compared with $x o p Q^{-} / \mathrm{pHM} 1: \operatorname{xop} Q$. The two predicted XopQ active site mutants (xop $Q^{-/ p H M 1:: x o p} Q^{D 116 A}$ and $x o p Q^{-} / \mathrm{pHM1}: \operatorname{xop} Q^{Y 279 A}$ ) exhibit a reduced hypersensitive response (HR) on Nicotiana benthamiana, a nonhost. However, Arabidopsis lines expressing either $x$ op $Q$ or $x o p Q^{Y 279 A}$ are equally proficient at suppression of LipA-induced callose deposition. Purified XopQ does not show NH activity on standard nucleoside substrates but exhibits ribose hydrolase activity on the nucleoside substrate analogue 4nitrophenyl $\beta$-D-ribofuranoside. The D116A and Y279A mutations cause a reduction in biochemical activity. These results indicate that mutations in the predicted active site of XopQ affect virulence and induction of the HR but do not affect suppression of innate immunity.
\end{abstract}

Plants have powerful innate immune responses that protect them against the vast majority of potential pathogens. These

GenBank accession numbers: KJ809115: type III effector XopQ from Xanthomonas oryzae pv. oryzae BXO43; KJ809116: D116A mutant of type III effector XopQ from Xanthomonas oryzae pv. oryzae BXO43; KJ809117: Y279A mutant of type III effector XopQ from Xanthomonas oryzae pv. oryzae $\mathrm{BXO} 43$.

Current address for Dipanwita Sinha: Singapore Centre on Environmental Life Sciences Engineering, Nanyang Technological University, Singapore 637551.

Corresponding author: R. V. Sonti; Telephone: +91-40-27192577; Fax: +91-40-27160591; E-mail: sonti@ccmb.res.in

* The $\boldsymbol{e}$-Xtra logo stands for "electronic extra" and indicates that three supplementary figures and two supplementary tables are published online.

(C) 2015 The American Phytopathological Society include a first layer of responses called pathogen-triggered immunity (PTI) which is induced upon recognition of microbeassociated molecular patterns such as flagellin, chitin, lipopolysaccharide, and so on, as well as damage-associated molecular patterns (DAMPs) such as cell wall degradation products. The suppression of PTI appears to be crucial for successful pathogenesis, and this is accomplished by secretion of a variety of microbial effectors directly into host cells. Plants have evolved a second layer of defense responses called effector-triggered immunity (ETI) that is involved in the recognition of the microbial effectors. ETI is often associated with a hypersensitive response (HR), which is a form of defense response associated with localized programmed cell death that is believed to serve in limiting the pathogen to the site of infection. Gram-negative phytopathogenic bacteria use the type 3 secretion system (T3SS) to inject type 3 effectors (T3E) directly into host cells, and several of these proteins are involved in suppression of host defense responses. These T3E, in turn, are recognized by intracellular receptors to mount a HR. The T3SS apparatus is encoded by hypersensitive response and pathogenicity ( $h r p$ ) genes which confer pathogenicity on host plants and elicit HRs in nonhost plants (Alfano and Collmer 1997).

Xanthomonas oryzae pv. oryzae causes bacterial blight, a serious disease of rice. The hrp gene cluster of this bacterium has been characterized and shown to be required for virulence (Zhu et al. 2000). Genome analysis has shown that the $X$. oryzae pv. oryzae genome potentially encodes genes for both transcription activator-like (TAL) and non-TAL types of T3E (Lee et al. 2005; Ochiai et al. 2005; Salzberg et al. 2008). X. oryzae pv. oryzae injects at least 16 non-TAL effectors into rice cells (Furutani et al. 2009; Song and Yang 2010). Previously, we have demonstrated that four $\mathrm{T} 3 \mathrm{E}$ of $X$. oryzae $\mathrm{pv}$. oryzae (namely, XopN, XopQ, XopX, and XopZ) are suppressors of cell wall damage (DAMP)-induced innate immunity in rice (Sinha et al. 2013). We have also demonstrated that a $x o p N$ $x o p Q x o p X$ xopZ quadruple mutant of $X$. oryzae pv. oryzae induces defense responses to the same extent as a T3S-deficient mutant in rice leaves, indicating that it is defective in suppression of PTI (Sinha et al. 2013). The XopZ, XopQ, XopX, $\mathrm{XopN}$, and XopR proteins are shown to be required for promoting full virulence of $X$. oryzae pv. oryzae on rice (Akimoto-Tomiyama et al. 2012; Sinha et al. 2013; Song and Yang 2010). There is a strain specificity with regard to the virulence phenotypes because mutations in the $x \circ p N$, xоp $Q$, and $x o p X$ genes of some $X$. oryzae pv. oryzae strains are fully virulence proficient (Song and Yang 2010; Zhao et al. 2013). 
This could be due to the presence of additional functions in these strains that are redundant with regard to the virulence promoting activities of XopN, XopQ, and XopX. The molecular mechanism by which these four Xop proteins suppress rice innate immunity and promote virulence is not known.

A xopN mutant of $X$. campestris pv. vesicatoria exhibits reduced growth on tomato (Kim et al. 2009) whereas an X. campestris pv. campestris 8004 xopN mutant exhibits reduced virulence in radish (Jiang et al. 2008). XopN from X. campestris pv. vesicatoria suppresses pathogen-associated molecular pattern-induced callose deposition and defense related gene expression in tomato and Arabidopsis plants (Kim et al. 2009). The XopN protein has been demonstrated to interact with tomato atypical receptor kinase 1 and 14-3-3 proteins in yeast as well as in planta. A xopX mutant of $X$. campestris pv. vesicatoria showed a partial virulence deficiency on host tomato and pepper plants whereas ectopic expression of the xopX gene in $N$. benthamiana resulted in suppression of host defense responses (Metz et al. 2005). The XopQ protein of X. euvesicatoria, a pathogen of pepper and tomato plants, has been shown to suppress ETI and promote growth in resistant host plants (Teper et al. 2014). Suppression of ETI occurs through interaction with a host 14-3-3 isoform called TFT4 that is involved in promoting ETI. XopQ has a mode I 14-3-3 binding motif, and mutation of a crucial serine residue in this motif affects binding to TFT4 and the ability to promote virulence.

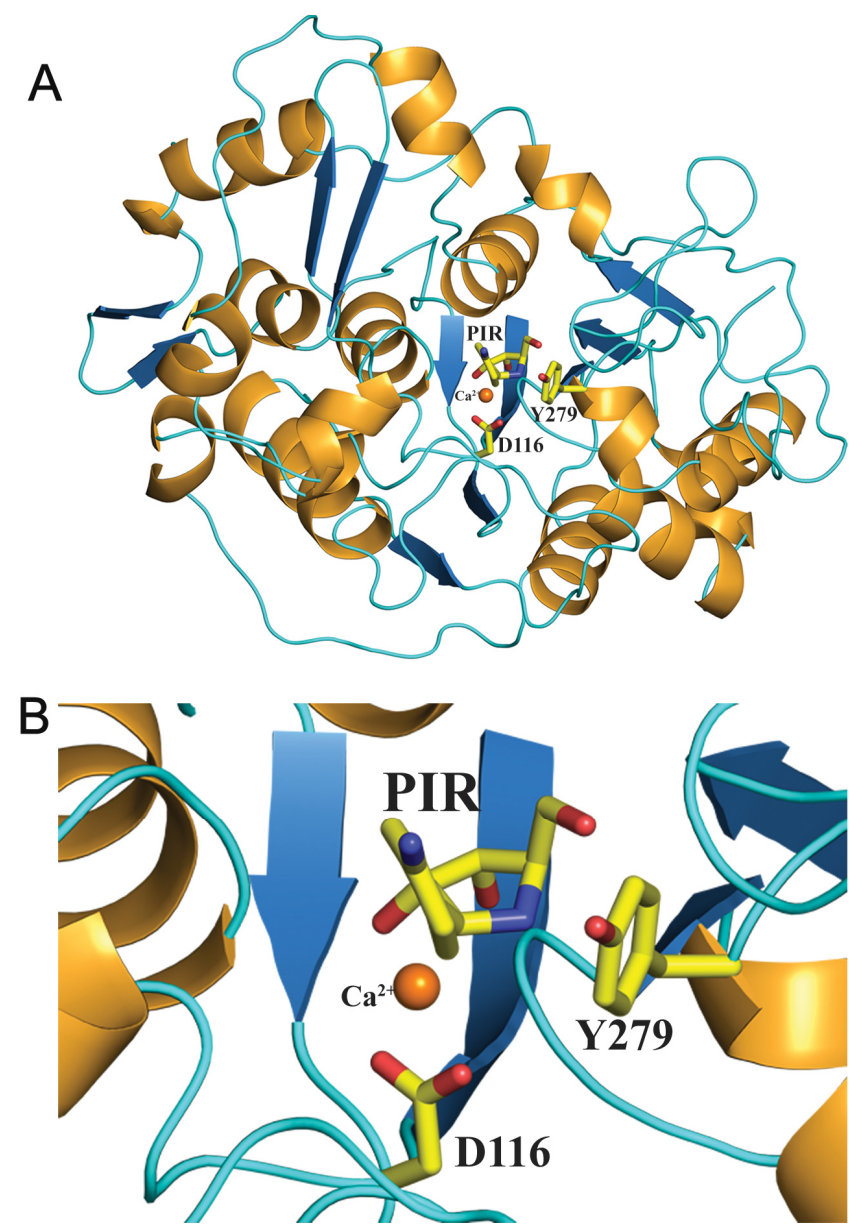

Fig. 1. Structural model of Xanthomonas oryzae pv. oryzae BXO43 XopQ. A, Structural model of BXO43 XopQ was developed using purine nucleoside hydrolase from Crithidia fasciculata (Protein Data Bank number: 2MAS). B, Putative catalytic residues D116 and Y279 along with the modeled inhibitor 2-(4-amino-phenyl)-5-hydroxymethyl-pyrrolidine-3, 4-diol (PIR) are depicted as sticks. The modeled $\mathrm{Ca}^{2+}$ ion located in the active site pocket of the enzyme is depicted in the dark orange sphere.
The XopQ protein is homologous to Hrp outer protein Q (HopQ1), a T3E of the model pathogen Pseudomonas syringae pv. tomato DC3000 that infects tomato and Arabidopsis. Transgenic tomato or Arabidopsis plants that express HopQ1 exhibit enhanced susceptibility to $P$. syringae pv. tomato. $P$. syringae pv. tomato induces a HR in the nonhost Nicotiana benthamiana. A HopQ1 mutant of $P$. syringae pv. tomato does not induce a $\mathrm{HR}$ and is able to cause disease on Nicotiana plants, indicating that HopQ1 is involved in restricting host range (Wei et al. 2007). HopQ1 has also been shown to interact with plant 14-33 proteins in a phosphorylation-dependent manner (Giska et al. 2013). A single amino acid substitution mutant of HopQ1 (HopQ1-S51A) that affects 14-3-3 binding affects virulence on tomato but does not affect the ability to induce a HR in Nicotiana spp. (Li et al. 2013b). The HopQ1 protein exhibits homology to nucleoside hydrolases $(\mathrm{NH})$ which cleave the N-glycosidic bond between the base and ribose sugar in nucleosides. Mutations in the NH domain that are predicted to affect catalytic activity are not effective in promoting virulence but retain the ability to induce a HR in Nicotiana spp. (Li et al 2013a). This suggests the possibility that biochemical activity of HopQ is necessary for promoting virulence but is not necessary for promoting a HR in Nicotiana spp. Transgenic Arabidopsis plants that express HopQ suppress flagellin-induced immune responses. Transgenic Arabidopsis plants that express HopQ1 variants that are mutated in conserved aspartate residues (which are predicted to be involved in nucleoside binding) are defective in the ability to suppress flagellin-induced PTI responses (Hann et al. 2014). Although HopQ1 is homologous to $\mathrm{NH}$ enzymes, the purified protein does not exhibit $\mathrm{NH}$ activity. Recently, it has been suggested that HopQ1 is a phosphoribohydrolase which acts on a cytokinin precursor, $\mathrm{N}^{6}$-isopentenyladenosine-5'-monophosphate (IPRMP), to release cytokinin (Hann et al. 2014).

$X$. oryzae pv. oryzae XopQ protein also exhibits limited homology to bacterial and protozoan NH. In this study, we have demonstrated that amino acid substitutions in the predicted active site of XopQ result in significant loss of virulence of $X$. oryzae pv. oryzae. These mutations also affect the ability of XopQ to induce a HR in the nonhost plant $N$. benthamiana. Surprisingly, the mutant versions of XopQ still retained most of the immune suppression activity of XopQ against PTI in rice and cell-wall damage-induced defense responses in Arabidopsis plants. Finally, we show that purified XopQ does not have NH activity when tested against a variety of nucleosides but exhibits limited ribose hydrolase activity when assayed on an NH substrate analogue, 4-nitrophenyl $\beta$-D-ribofuranoside (pNPR). Compared with wild-type XopQ protein, the mutant versions of XopQ exhibit reduced ribose hydrolase activity against pNPR. Overall, the results suggest that mutations in the predicted active site of XopQ affect virulence and the ability to induce a HR in $N$. benthamiana but either do not affect or have mild effects on the ability to suppress host immune responses.

\section{RESULTS}

\section{X. oryzae pv. oryzae XopQ exhibits sequence similarity to $\mathrm{NH}$.}

X. oryzae pv. oryzae $\mathrm{BXO} 43$ (our laboratory wild-type strain) XopQ (GenBank accession number: KJ809115) shares amino acid identity with $X$. oryzae pv. oryzae strains MAFF31108 (99\%), KACC10331 (99\%), and PXO99A (99\%); X. axonopodis pv. citri 306 (93\%); X. campestris pv. campestris strains 8004 (64\%) and ATCC33913 (64\%); and X. campestris pv. vesicatoria $85-10$ (94\%). XopQ has $61 \%$ amino acid identity with HopQ1-1 of $P$. syringae pv. tomato DC3000 
(Yu et al. 2013). The BXO43 XopQ is a 464-amino-acid protein predicted to have similarity to inosine-uridine $\mathrm{NH}$ in several organisms (for example, Escherichia coli, Bacillus anthracis, and protozoan parasite Crithidia fasciculata (Supplementary Table S1). NH catalyze the cleavage of the N-glycosidic bond of nucleosides to release a ribose sugar and the respective base. A DXDXXXDD motif in the $\mathrm{N}$-terminal region of the protein is the hallmark of typical $\mathrm{NH}$ (Versées and Steyaert 2003). However, X. oryzae pv. oryzae XopQ possesses this signature motif with a variation (DXXXDXDD). This same variation is also found in the signature motif in $P$. syringae pv. tomato HopQ1. A homology model of XopQ was prepared using SWISS-MODEL in the automated mode (Fig. 1). Subsequent structural analysis was performed with the crystal structure of purine NH from $C$. fasciculata (Protein Data Bank [PDB] number: 2MAS). Previous studies have suggested an acid-base hydrolysis in which an aspartate from the motif is involved in catalysis of NH (Degano et al. 1996, 1998; Giabbai and Degano 2004). The model of XopQ further suggests that the tyrosine 279 is in close vicinity to the putative active site along with the aspartates from the motif. Therefore, putative active site residues $x o p Q^{D 116}$ (which is part of the motif) and $x o p Q^{Y 279}$ were selected to perform mutational studies.

Recently, the crystal structure of XopQ protein encoded by XOO4466 of X. oryzae pv. oryzae KACC10331 has been solved which had the same amino acid sequence of XopQ (BXO43), except for amino acid substitution at position $83(\mathrm{H}$ with V) and position 309 (A with G) (Yu et al. 2013). Based on this crystal structure, the selected residues (xop $Q^{D 116}$ and xop $Q^{Y 279}$ ) were positioned in the same putative catalytic active site (Supplementary Fig. S1).

\section{Mutations in the putative catalytic active site} $\left(x o p Q^{D 116 A}\right.$ and $\left.x o p Q^{Y 279 A}\right)$ of $X$. oryzae pv. oryzae XopQ affect virulence on rice.

A XopQ disruption mutation in the genetic background of the $X$. oryzae pv. oryzae $\mathrm{BXO} 43$ strain was earlier shown to cause virulence deficiency (Sinha et al. 2013). The role of putative active site residues in promoting virulence was studied by assessing virulence phenotypes of the aspartic acid mutant of $x o p Q\left(x o p Q^{-} / \mathrm{pHM} 1:: x o p Q^{D 116 A}\right)$, the tyrosine mutant of $x o p Q$ $\left(x o p Q^{-} / \mathrm{pHM} 1:: x o p Q^{Y 279 A}\right)$, and the double mutant (xop $Q^{-}$ /pHM1::xop $Q^{D 116 A Y 279 A}$ ) in comparison with wild-type $x o p Q$ $\left(x o p Q^{-} / \mathrm{pHM} 1:: x o p Q\right)$ and the vector control (xop $\left.Q^{-} / \mathrm{pHM} 1\right)$. Virulence phenotypes were determined by clip inoculating rice leaves and assessing lesion lengths 10 days after inoculation. A $x o p Q$ mutant showed significantly reduced lesion lengths when compared with those produced by the wild type. In trans, introduction of the $x o p Q$ gene complemented the virulence-deficient phenotype of the $x o p Q$ mutant. The substitution of aspartate at position 116 with alanine (D116A) and tyrosine at position 279 with alanine (Y279A) in XopQ protein ( $x \circ \mathrm{p}^{-} /$ pHM1::xop $Q^{D 116 A}, \quad x o p Q^{-} / \mathrm{pHM} 1: x_{0 o p} Q^{Y 279 A}$, and $x o p Q^{-} /$ pHM1::xop $Q^{D 116 A Y 279 A}$ ) affected the ability to restore virulence to the levels obtained following complementation with the wild-type allele of $x o p Q$ (Fig. $2 \mathrm{~A}$ and $\mathrm{B}$ ). In pairwise comparisons of wild-type $x o p Q$ with each of the single and double amino acid substitution mutants, the differences were statistically significant.

The bacterial counts in the inoculated leaves were also estimated immediately after inoculation and 6 days after inoculation. A $x o p Q$ mutant showed significantly reduced growth yield in rice leaves when compared with the wild type strain. Introduction of the $x o p Q$ gene in trans complemented the in planta growth defect (Fig. 3). The D116A and Y279A substitutions in the XopQ protein $\left(x o p Q^{-} / \mathrm{pHM} 1: x_{0 p} Q^{D 116 A}\right.$, xop $Q^{-}$ $/ \mathrm{pHM} 1:: x o p Q^{Y 279 A}$, and $x o p Q^{-} / \mathrm{pHM} 1:: x o p Q^{D 116 A Y 279 A)}$ affected
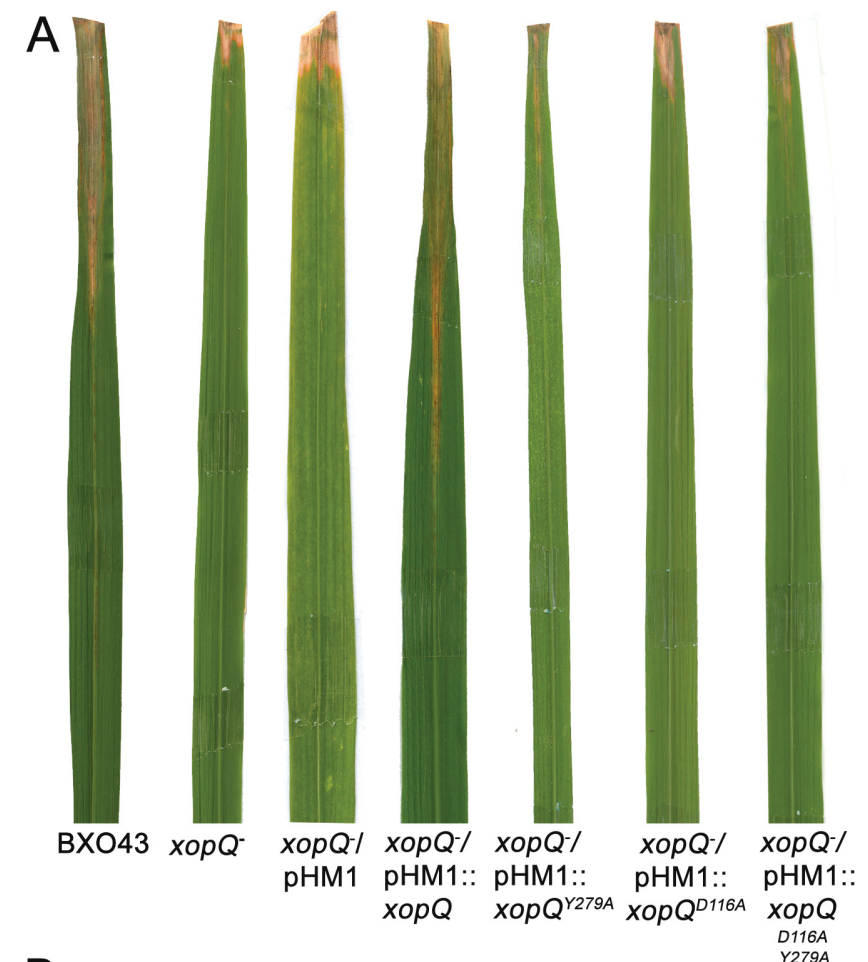

B

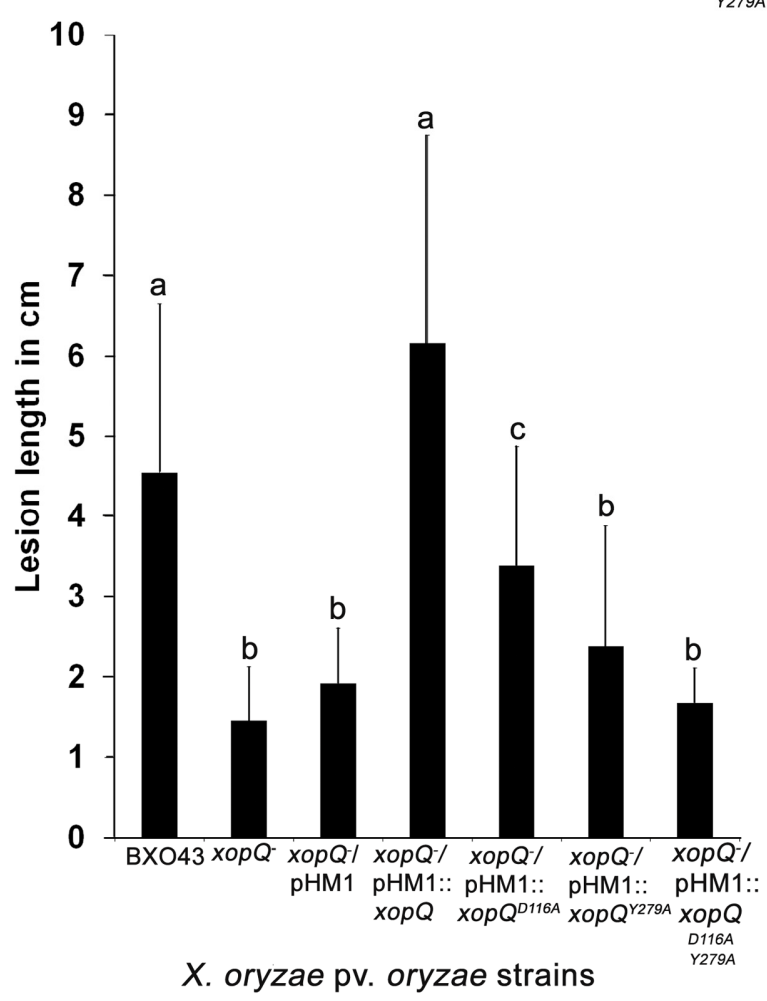

Fig. 2. Mutations in the predicted active site of Xanthomonas oryzae pv. oryzae XopQ affect virulence on rice. Leaves of susceptible rice 'Taichung-Native 1' (TN-1) were clip inoculated with the following $X$. oryzae pv. oryzae strains: BXO43 (wild type), xopQ mutant, $x$ op $Q^{-} / \mathrm{pHM} 1:: x o p Q$ (complemented strain), xop $Q^{-} / \mathrm{pHM} 1:: x \circ p Q^{Y 279 A}$, xоp $Q^{-} / \mathrm{pHM} 1:: x o p Q^{D 116 A}$, $x o p Q^{-} / \mathrm{pHM} 1: x_{0 p} Q^{D 116 A} Y 279 A$, and $x o p Q^{-} / \mathrm{pHM} 1$ (vector control). A, Virulence phenotype on rice leaves. Leaves were photographed 10 days after inoculation. B, Lesion lengths were measured 10 days after inoculation. Error bars indicate the standard deviation of readings from 17 inoculated leaves. Columns in plots capped with the same letter were not significantly different based on analysis of variance and comparisons for all done using the Tukey-Kramer honestly significance difference test $(P<$ $0.05)$. The experiment was repeated several times with similar results. Similar results were obtained in independent experiments. 
the ability to complement the in planta growth defect of the $x o p Q$ mutant (Fig. 3). Western blot analysis using anti-XopQ polyclonal antibodies indicated that the D116A, Y279A (single amino acid substitution), and D116A Y279A (double amino acid substitution) mutant proteins are expressed to almost the same extent as the wild-type XopQ protein in X. oryzae pv. oryzae strains growing within rice leaves (Supplementary Fig. $\mathrm{S} 2$ ). Overall, these results indicate that the mutated residues in XopQ protein are required for promoting virulence of $X$. oryzae pv. oryzae on rice.

The HR elicited by $X$. oryzae pv. oryzae in the nonhost plant $N$. benthamiana is attenuated in the predicted XopQ catalytic active site mutants $x o p Q^{D 116 A}$ and $x o p Q^{Y 279 A}$.

Infiltration of wild-type $X$. oryzae pv. oryzae into $N$. benthamiana induces a HR in the infiltrated zone as measured by uptake of trypan blue, whereas a T3S-deficient strain of $X$. oryzae pv. oryzae (mutated for the $h r p B 6(h r c N)$ gene) (Jha et al. 2007) displays no HR (Fig. 4A). The $x o p Q$ mutant is defective in inducing the HR when infiltrated in $N$. benthamiana leaves. The complemented strain (xop $Q^{-} / \mathrm{pHM} 1:$ :xop $\left.Q\right)$ regains the ability to elicit a $\mathrm{HR}$ while the vector control (xop $Q^{-}$ /pHM1) does not. This indicates that the XopQ protein of $X$. oryzae pv. oryzae induces a HR in $N$. benthamiana. The $X$. oryzae pv. oryzae XopQ mutant complemented with single amino acid substitution mutants (xop $Q^{-} / \mathrm{pHM} 1: x \circ \mathrm{xp} Q^{D 116 A}$ and $\left.x o p Q^{-} / \mathrm{pHM} 1:: x o p Q^{Y 279 A}\right)$ and the double amino acid substitution mutant (xop $Q^{-} / \mathrm{pHM} 1: x \operatorname{xop} Q^{D 116 A}$ Y279A $)$ were checked for their ability to induce a HR in N. benthamiana. The ability to induce a HR was significantly attenuated by these mutations

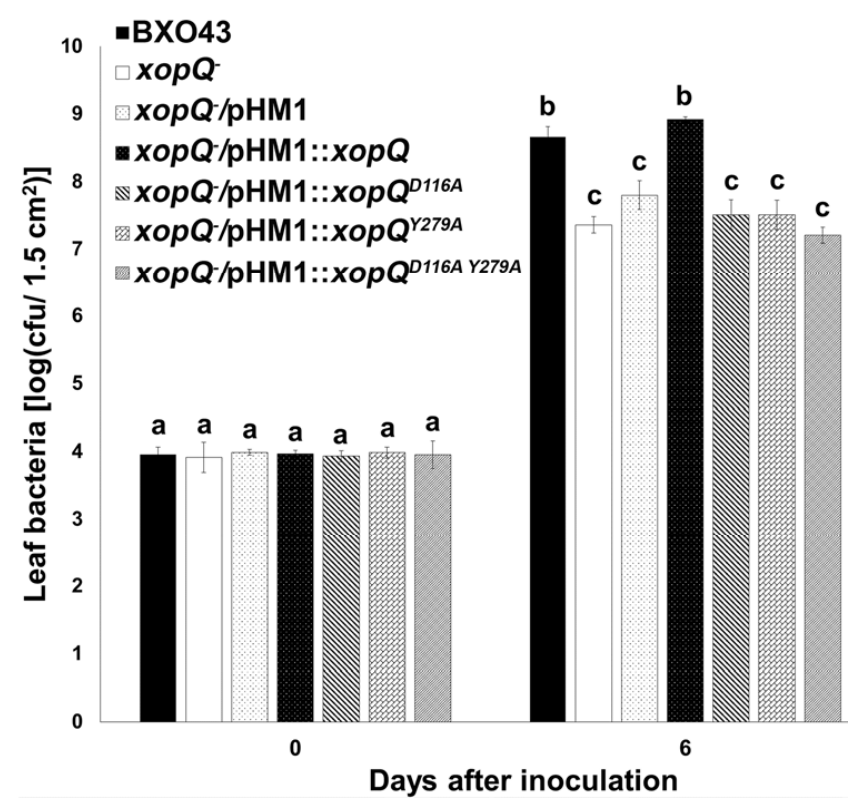

Fig. 3. Mutations in the predicted active site of Xanthomonas oryzae pv. oryzae XopQ affect bacterial growth in rice leaves. Leaves of susceptible rice 'Taichung-Native 1' (TN-1) were clip inoculated with cultures $\left(10^{8}\right.$

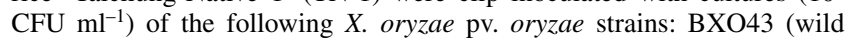
type), $x o p Q$ mutant, $x o p Q^{-} / \mathrm{pHM} 1: x \circ \mathrm{p} Q$ (complemented strain), $x o p Q^{-}$ $/ \mathrm{pHM} 1:$ :xop $Q^{Y 279 A}, \quad$ xоp $Q^{-} / \mathrm{pHM} 1:: x$ op $Q^{D 116 A}, \quad$ xор $Q^{-} / \mathrm{pHM} 1:: x o p Q^{D 116 A}$ ${ }_{Y 279 A}$, and $x \circ p Q^{-} / \mathrm{pHM} 1$ (vector control). Bacterial cell numbers were estimated in $1.5-\mathrm{cm}$ leaf pieces cut from the end of each clipped leaf at 0 and 6 days after inoculation. Error bars indicate the standard deviation of populations measured from three leaves/treatment. Columns within each plot capped with the same letter were not significantly different based on analysis of variance and comparisons for all were done using the TukeyKramer honestly significance difference test $(P<0.05)$. The experiment was repeated twice with similar results.
(Fig. 4A). The $X$. oryzae pv. oryzae xop $Q$ mutant complemented by the double amino acid substitution mutant version of XopQ $\left(x o p Q^{-} / \mathrm{pHM} 1:: x o p Q^{D 116 A}{ }^{Y 279 A}\right)$ was a weaker inducer of the HR than the $X$. oryzae pv. oryzae xop $Q$ mutant complemented with the single amino acid substitution mutants (xop $Q^{-} / \mathrm{pHM} 1:$ : $x o p Q^{D 116 A}$ and $x o p Q^{-} / \mathrm{pHM} 1:: x o p Q^{Y 279 A}$ ) (Fig. 4A). Taken together, this suggests that the mutated residues of XopQ are important for induction of the HR in the nonhost plant $N$. benthamiana.

Leakage of electrolytes occurs during the HR. An estimate of the leaked electrolytes can provide a quantitative measure of the extent of the HR occurring in leaves. We measured the electrolyte leakage from $N$. benthamiana leaves infiltrated with the wild type (BXO43), xopQ mutant, xopQ mutant with
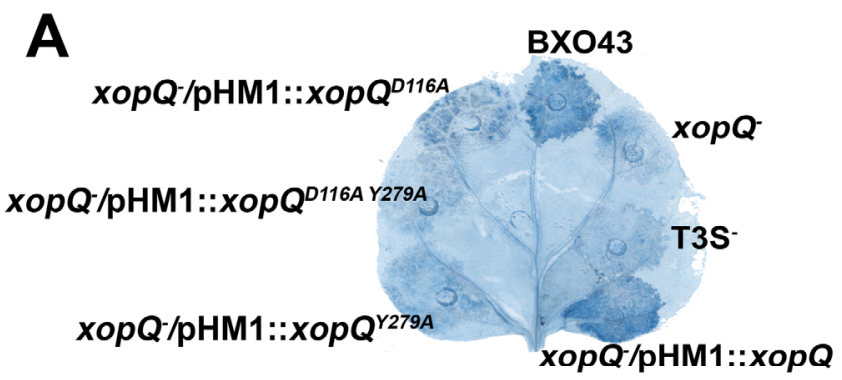

B

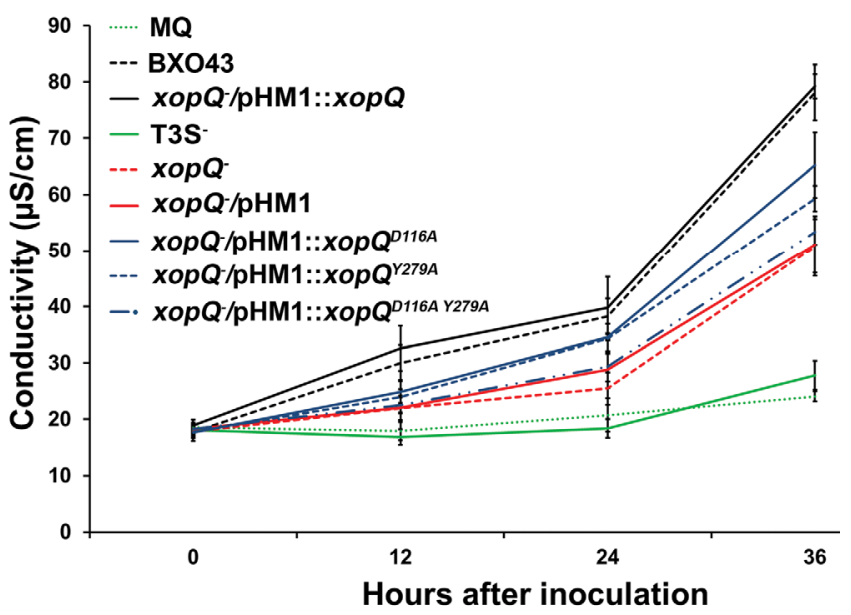

Fig. 4. Effect of mutations in the predicted active site residues of Xanthomonas oryzae pv. oryzae XopQ protein on induction of a hypersensitive response (HR) in Nicotiana benthamiana. Leaves of $N$. benthamiana were infiltrated with the following strains: wild type (BXO43), type 3 secretionnegative $\left(\mathrm{T}^{-} \mathrm{S}^{-}\right)$mutant, xop $Q$ mutant, xop $Q^{-} / \mathrm{pHM} 1:$ :xop $Q$ (complemented strain), xop $Q^{-} / \mathrm{pHM} 1:: \operatorname{xop} Q^{Y 279 A}$, xop $Q^{-} / \mathrm{pHM} 1:: x o p Q^{D 116 A}$, xop $Q^{-}$ /pHM1::xop $Q^{D 116 A Y 279 A}$, and $x o p Q^{-} / \mathrm{pHM} 1$ (vector control). A, Cell death was visualized by trypan blue staining of the same leaf. BXO43 and the complemented strain induced a HR in $N$. benthamiana leaves; the XopQ mutant, vector control, and $\mathrm{T}_{3} \mathrm{~S}^{-}$strains did not induce the HR. The D116A and Y279A single mutants and the D116A Y279A double mutant also affected the ability to induce a HR. The experiment was repeated twice with similar results. B, Electrolyte leakage was measured in the harvested leaves at different time points after infiltration. Statistically significant differences were obtained using analysis of variance and comparisons done using the Tukey-Kramer honestly significance difference test $(P<$ 0.05 ) from $N$. benthamiana leaves infiltrated with either BXO43 or $x o p Q^{-}$/ pHM1::xopQ (complemented strain) compared with leaves treated with xop $Q$ mutant, xop $Q^{-} / \mathrm{pHM} 1$, xop $Q^{-} / \mathrm{pHM} 1: x_{0} Q^{D 116 A}$, xop $Q^{-} / \mathrm{pHM} 1:$ : xop $Q^{Y 279 A}$, and xop $Q^{-} / \mathrm{pHM} 1: x_{0 p} Q^{D 116 A}{ }^{279 A}$. Statistically significant differences were also observed in the comparison of either xop $Q$ mutant or $x o p Q^{-} / \mathrm{pHM} 1$ to $x$ op $Q^{-} / \mathrm{pHM} 1:$ xop $Q^{D 116 A}$. However, statistically significant differences were not observed in the following comparisons: $x$ op $Q^{-}$/ pHM1 with $x$ op $Q^{-} / \mathrm{pHM} 1:$ xop $Q^{Y 279 A}$ or $x$ op $Q^{-} / \mathrm{pHM} 1: x_{0 p} Q^{D 116 A}$ Y279A. Experiments were repeated three times with similar results. 
complementing clone (xop $Q^{-} / \mathrm{pHM} 1: x$ : $\left.x p Q\right)$, vector control (xop $Q^{-} / \mathrm{pHM} 1$ ), xopQ mutant complemented with xopQ single amino acid substitution mutants (xop $Q^{-} / \mathrm{pHM} 1:: x \circ p Q^{D 16 A}$ and $x o p Q^{-} / \mathrm{pHM} 1:$ xop $Q^{Y 279 A}$ ), and $x o p Q$ mutant complemented with the double amino acid substitution mutant (xop $Q^{-}$ /pHM1::xop $Q^{D 116 A Y 279 A}$ ). We found that infiltration of the wildtype strain results in significant amounts of ion leakage $36 \mathrm{~h}$ after infiltration. Minimal leakage of electrolytes is observed in leaves infiltrated with either water or a $\mathrm{T}_{3} \mathrm{~S}^{-}$mutant. As compared with the wild-type strain, infiltration with a $x o p Q$ mutant results in significantly reduced amounts of leaked electrolytes. Introduction of a clone containing the wild-type $x o p Q$ gene restores electrolyte leakage to the levels induced by the wild-type strain (Fig. 4B). Introduction of clones containing single and double amino acid substitutions in XopQ results in reduced levels of electrolyte leakage compared with the wildtype strain, again indicating that the amino acid substitutions compromise the ability of the XopQ protein to elicit a HR in $N$. benthamiana. It is to be noted that the $\operatorname{xop} Q$ knockout mutant triggers more leakage of electrolytes than theT3S-deficient mutant, indicating that there must be another T3S effector besides XopQ that is involved in triggering ion leakage.

\section{Effect of $x o p Q^{D 116 A}$ and $x o p Q^{Y 279 A}$ mutations \\ of $X$. oryzae pv. oryzae XopQ}

on suppression of innate immunity in rice.

Callose deposition is a well-known marker of PTI (Jha et al 2007; Nicaise et al. 2009). We have previously shown that, in Agrobacterium-mediated transient transfer assays, the XopN, XopQ, XopX, and XopZ proteins suppress lipase/esterase A (LipA)-induced callose deposition in rice (Sinha et al 2013). A quadruple mutant that is mutated in genes for all four Xop proteins induces callose deposition similar to an $X$. oryzae pv. oryzae T3S mutant (Sinha et al. 2013). The property of the T3S-defective mutant, as well as the xopN xop $Q$ xopX xopZ quadruple mutant, to trigger callose deposition is attributed to their failure to suppress PTI. In the present study, we showed that constitutive plasmid-borne expression of the $x o p Q$ gene in this quadruple mutant background was sufficient to suppress rice innate immunity as assessed by reduced callose deposition in leaves (Fig. 5A and B). To assess effects of XopQ active site mutations in suppression of quadruple mutant-induced callose deposition, rice leaves were infiltrated with the $X$. oryzae pv. oryzae quadruple mutant harboring $\mathrm{pHM} 1, \mathrm{pHM} 1:: x o p Q$, pHM1::xop $Q^{D 116 A}$, pHM1::xop $Q^{Y 279 A}$, and $\mathrm{pHM1::xop} Q^{D 116 A}$ ${ }_{Y 279 A}$ constructs. Our expectation was that, if the mutated amino acid residues of XopQ are required for suppression of rice innate immunity, the amino acid substitution mutants would be defective in suppression of callose deposition induced by the quadruple mutant. The results indicate that each of the mutant XopQ proteins is capable of suppressing PTI, although the xop $Q^{D 116 A}$ mutant does show a reduced efficiency in suppression of PTI compared with wild-type XopQ (Fig. 5A and B). Importantly, the $x o p Q^{D 116 A Y 279 A}$ double mutant is as capable as wild-type $x o p Q$ at suppression of PTI.

We next proceeded to test whether a mutation in the predicted active site of XopQ would be required for suppression of LipAinduced innate immunity. For this purpose, we generated transgenic Arabidopsis plants which exhibit estradiol-inducible expression of either wild-type $x o p Q$ or $x o p Q^{Y 279 A}$. We chose the Y279A mutation for this assay because of the stronger phenotype of this mutation compared with D116A in the virulence (Fig. 2) and HR assays (Fig. 4). In the absence of estradiol, LipA induces callose deposition in Arabidopsis plants that are transgenic for either wild-type $x o p Q$ or $x o p Q^{Y 279 A}$ (Fig. 6A and B). In the presence of estradiol, LipA-induced callose deposition is suppressed in transgenic plants expressing either wild-type

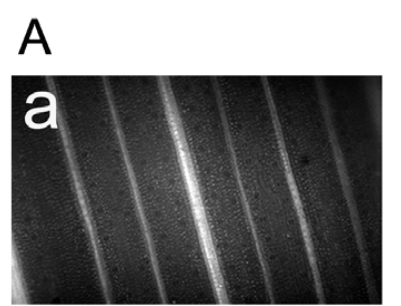

\section{Buffer}

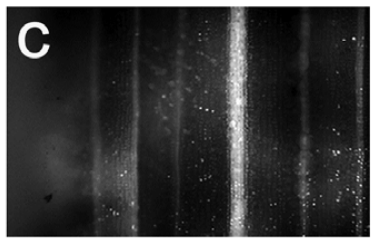

T3S- $^{-}$

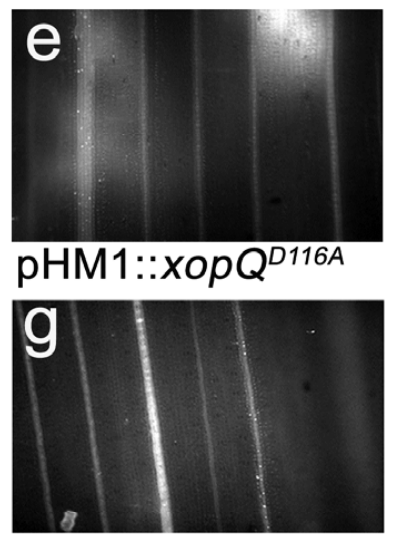

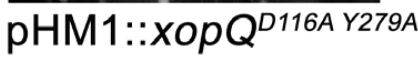

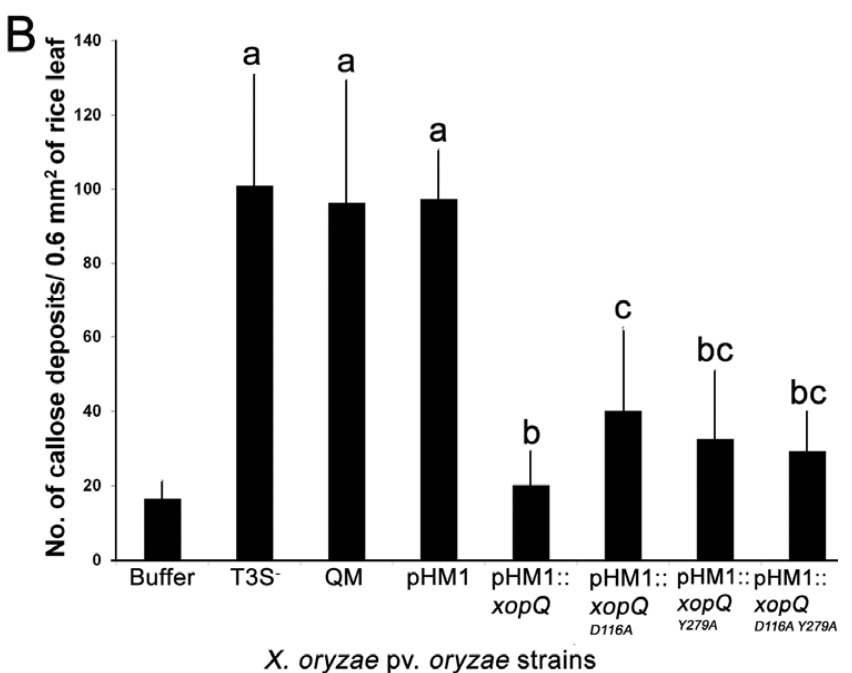

Fig. 5. Effect of mutations in predicted active site residues on suppression of pathogen-triggered innate immunity in rice leaves by XopQ. A, Rice leaves were infiltrated with one of the following: a, buffer; b, xоpN xоpQ xopX xopZ quadruple mutant (QM); c, T3S ${ }^{-}$; and quadruple mutant harboring the following constructs: d, pHM1:xop $Q ; \mathbf{e}$, pHM1::xop $Q^{D 116 A} ; \mathbf{f}$, pHM1::xop $Q^{Y 279 A} ; \mathbf{g}$, pHM1::xop $Q^{D 116 A}$ Y279A; and h, pHM1. The leaves were subsequently stained $20 \mathrm{~h}$ later with aniline blue and visualized under an epifluorescence microscope. Callose deposition is seen as white spots in the figures. B, Mean and standard deviation were calculated for number of callose deposits observed in an area of $0.60 \mathrm{~mm}^{2}$. Data were collected from seven leaves per treatment in each experiment. Columns in a plot capped with the same letter were not significantly different based on analysis of variance and comparisons for all done using the Tukey-Kramer honestly significance difference test $(P<0.05)$. The experiment was repeated several times with similar results. 

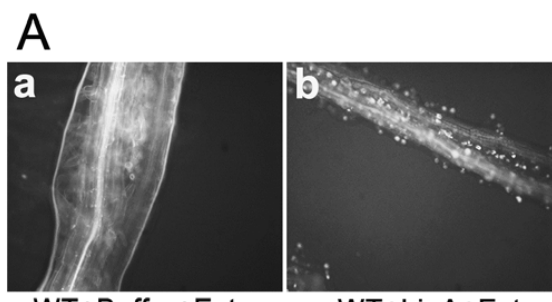

WT+Buffer+Est
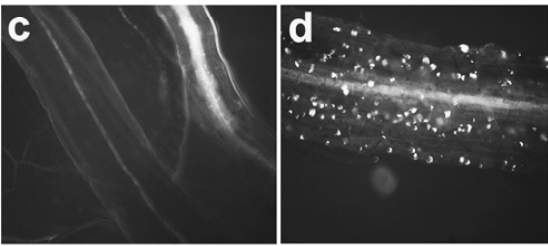

$x o p Q+$ LipA
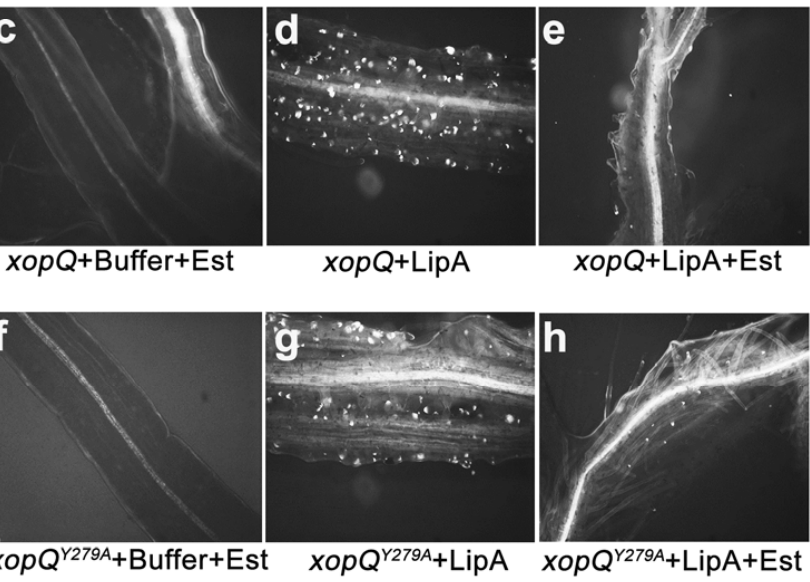

B

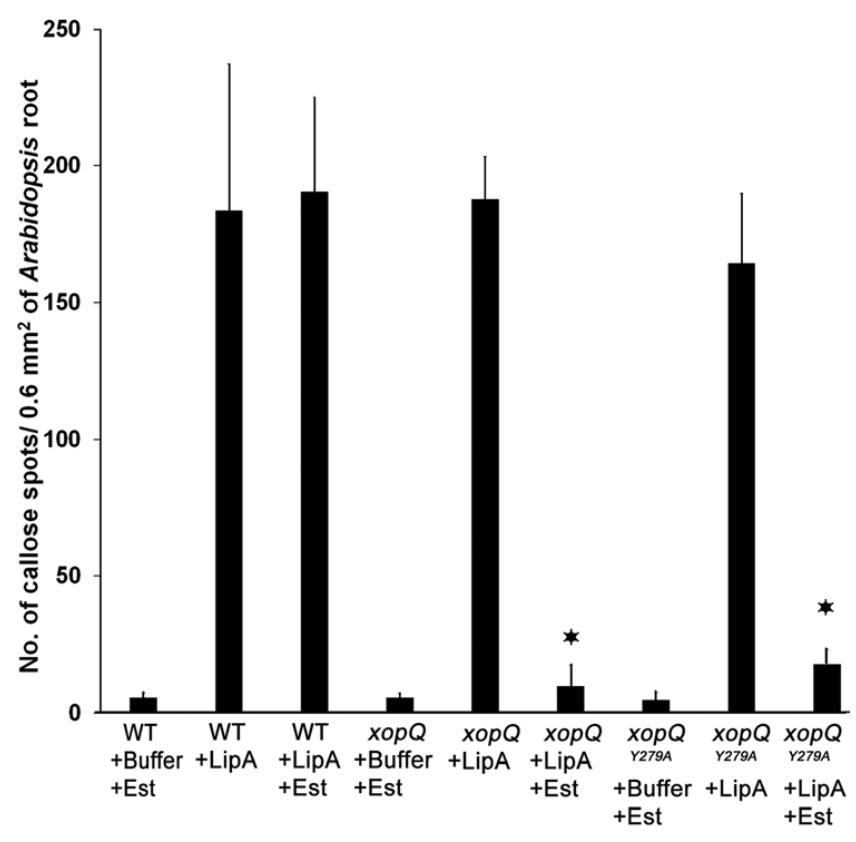

Fig. 6. Transgenic expression of Xanthomonas oryzae pv. oryzae XopQ in Arabidopsis suppresses cell wall damage-induced callose deposition. Arabidopsis thaliana plants of the following three genotypes were used: wild type Col-0 (WT) and transgenic plants carrying either xop $Q$ or $x_{x o p} Q^{Y 279 A}$. A, a, WT + buffer + estradiol (Est); b, WT + lipase/esterase A (LipA) + Est; c, xop $Q+$ buffer + Est; d, xop $Q+$ LipA; e, xop $Q+$ LipA + Est; f, xop $Q^{Y 279 A}+$ buffer + Est; $\mathbf{g}$, xop $Q^{Y 279 A}+\mathrm{LipA}$; and h, xop $Q^{Y 279 A}+$ LipA + Est. Roots were subsequently stained with aniline blue and visualized under an epifluorescence microscope. B, Callose deposits were quantified from $0.60-\mathrm{mm}^{2}$ area of roots. Data were collected from eight roots in each experiment and two to three different viewing areas from each root. Data were analyzed using analysis of variance and comparisons was done using the Tukey-Kramer honestly significance difference test $(P<0.05)$. Asterisks above the bars indicate significant differences relative to the corresponding uninduced lines, respectively. Statistically significant differences were not observed in the following comparisons: xop $Q+$ LipA with xop $Q^{Y 279 A}+$ LipA or $x o p Q+$ LipA + Est with $x o p Q^{Y 279 A}+$ LipA + Est. Similar results were obtained in three independent experiments. $x o p Q$ or $x o p Q^{Y 279 A}$. Estradiol by itself does not suppress LipAinduced callose deposition in Arabidopsis plants that are not transgenic for $x o p Q$ (M. K. Gupta and R. Sonti, unpublished data). These results indicate that the Y279A mutation does not affect the ability of XopQ protein to suppress LipA-induced callose deposition in Arabidopsis plants and is consistent with our observation (Fig. 5A and B) that the $x o p Q^{Y 279 A}$ mutation does not affect the ability of XopQ to suppress rice innate immunity.

\section{X. oryzae pv. oryzae XopQ protein has pNPR-mediated ribose hydrolase activity which is affected by the $x o p Q^{D 116 A}$ and $x o p Q^{Y 279 A}$ mutations.}

Wild-type XopQ protein and the two substitution mutants were overexpressed in E. coli and purified. To determine whether XopQ has NH activity, standard substrates such as inosine, guanosine, thymidine, cytidine, and uridine were used in activity assays (as described below). We did not observe any $\mathrm{NH}$ activity on these standard substrates. Consistent with these results, in recent studies, two independent groups have found that HopQ1-1 does not exhibit NH activity on standard substrates (Li et al. 2013a). However, we observed a weak biochemical activity for XopQ using a chromogenic NH substrate analogue pNPR. NH enzymes are known to cleave pNPR and release ribose and $p$-nitrophenol, a colored product. The biochemical activity of the wild-type and mutant proteins was quantitated using pNPR as a substrate (as described below). $K m$ values were $334.73 \pm 20.0 \mu \mathrm{M}$ for $\mathrm{XopQ}, 414.74 \pm 26.6$ $\mu \mathrm{M}$ for $\mathrm{XopQ}^{\mathrm{Y} 279 \mathrm{~A}}$, and $600.97 \pm 50.8 \mu \mathrm{M}$ for $\mathrm{XopQ}^{\mathrm{D} 116 \mathrm{~A}}(\mathrm{Ta}-$ ble 1). By comparison, NH from Trypanosoma brucei brucei has a $K m$ value of $562 \pm 51 \mu \mathrm{M}$ for pNPR (Parkin 1996). These data indicate that the affinity of wild-type XopQ protein for substrate is higher than the mutant proteins. The Kcat for $\mathrm{XopQ}, \mathrm{XopQ}^{\mathrm{D} 116 \mathrm{~A}}$, and $\mathrm{XopQ} \mathrm{Q}^{\mathrm{Y} 279 \mathrm{~A}}$ was $0.000277,0.000271$, and $0.000148 \mathrm{~s}^{-1}$, respectively, indicating a very poor activity of XopQ toward pNPR. The Kcat/Km for XopQ, XopQ ${ }^{\mathrm{D} 116 \mathrm{~A}}$, and $\mathrm{XopQ}{ }^{\mathrm{Y} 279 \mathrm{~A}}$ was found to be $0.83,0.45$, and $0.35 \mathrm{M}^{-1} \mathrm{~s}^{-1}$, respectively (Table 1). This indicates that the mutant proteins have less catalytic efficiency than the wild-type protein when pNPR is used as substrate. Taken together, these results suggest that XopQ possesses a ribose hydrolase activity with very poor activity on pNPR and suggest that the mutants have reduced affinity and catalytic efficiency toward this substrate.

\section{DISCUSSION}

In this study, homology modeling was used to predict the structure of $X$. oryzae pv. oryzae XopQ protein. Based on this model, the D116 and Y279 residues were predicted to be near the active site of the protein. The crystal structure of XopQ, which was described recently (Yu et al. 2013), confirms that these residues are indeed at the predicted active site of XopQ. The XopQ ${ }^{\text {D116A }}, X$ opQ ${ }^{\text {Y279A }}$, and XopQ ${ }^{\text {D116A Y279A }}$ mutants were deficient for virulence on rice and the ability to induce a HR in the nonhost plant, $N$. benthamiana. In a recent study, predicted

Table 1. Kinetic parameters of ribose hydrolase like activity associated with Xanthomonas oryzae pv. oryzae XopQ and XopQ mutants ${ }^{\mathrm{a}}$

\begin{tabular}{lccc}
\hline Parameters & $\boldsymbol{x o p} \boldsymbol{Q}$ & $\boldsymbol{x o p} \boldsymbol{Q}^{\text {Y279A }}$ & $\boldsymbol{x o p}^{\text {DI16A }}$ \\
\hline$K m(\mu \mathrm{M})$ & 334.73 & 414.74 & 600.97 \\
$K c a t\left(\mathrm{~s}^{-1}\right)$ & 0.000277 & 0.000148 & 0.000271 \\
$K c a t / K m\left(\mathrm{M}^{-1} \mathrm{~s}^{-1}\right)$ & 0.822 & 0.345 & 0.448 \\
\hline
\end{tabular}

a Assays were performed with overexpressed and purified proteins from Escherichia coli along with 4-nitrophenyl $\beta$-D-ribofuranoside substrate. Absorption of the product was determined spectrophotometrically at 405 nm. Km, Kcat, and Kcat/Km values were calculated using the MichaelisMenten equation (Cleland 1979). 
NH-deficient mutants of HopQ1 are reported to be deficient in promoting virulence but are proficient at inducing a HR in nonhost Nicotiana plants (Li et al. 2013a). It is puzzling that predicted NH-deficient mutants of HopQ1 are proficient at inducing a HR in Nicotiana spp. while the predicted ribose hydrolase-deficient mutants of XopQ are deficient at inducing the HR. It is possible that, in addition to promoting enzyme activity, the D116 and Y279 residues are involved in promoting protein-protein interactions with some Nicotiana proteins that are required for triggering the HR.

The XopQ ${ }^{\mathrm{D} 116 \mathrm{~A}}$ mutation had a moderate effect on the ability of XopQ to suppress PTI in rice. The XopQ ${ }^{\mathrm{Y} 279 \mathrm{~A}}$ mutant and the $\mathrm{XopQ}^{\mathrm{D} 116 \mathrm{~A}-\mathrm{Y} 279 \mathrm{~A}}$ double mutant were as proficient as the wild-type protein at suppressing PTI in rice. Inducible overexpression of either XopQ or XopQ ${ }^{\mathrm{Y} 279 \mathrm{~A}}$ resulted in suppression of cell-wall-degrading enzyme-induced immune responses in transgenic Arabidopsis lines. These results suggest that biochemical activity of XopQ might not be required for suppression of PTI in either rice or Arabidopsis. In contrast, a predicted NH-deficient mutant of HopQ1 is defective in suppression of PTI in Arabidopsis (Hann et al. 2014). This suggests that biochemical activity of HopQ1 but not XopQ may be required for suppression of PTI. If biochemical activity of $X$. oryzae pv. oryzae XopQ is not required for suppression of host innate immunity, what is the attribute of this protein that might contribute to suppression of PTI? The ability of XopQ to interact with 14-3-3 proteins may be crucial for suppression of host innate immunity. Teper and associates (2014) have shown that $X$. campestris pv. vescicatoria XopQ protein suppresses ETI in pepper by interacting with specific host 14-3-3 proteins through a mode I binding motif. Mutation of a serine residue (S65) within this motif to alanine results in loss of interaction with the 14-3-3 protein and an inability to suppress ETI. The X. oryzae pv. oryzae XopQ protein has a similar mode I 14-3-3 binding motif and it is possible that this protein might be suppressing PTI in rice by binding to 14-3-3 proteins.

In addition to suppressing PTI in rice, the $X$. oryzae pv. oryzae XopQ protein was found to suppress cell wall damage-induced callose deposition in Arabidopsis roots. The fact that XopQ can suppress innate immunity in a monocot and a dicot plant indicates that it has a broad host range for suppression of innate immunity and shows that the plant components that are targeted by XopQ are well conserved across the monocot and dicot divide. These conserved host components may be 14-3-3 proteins. It remains to be determined whether $X$. oryzae pv. oryzae XopQ protein binds to 14-3-3 proteins and whether this activity has a role in suppression of PTI in rice and Arabidopsis.

$\mathrm{NH}$ hydrolyze the $\mathrm{N}$-glycosidic bond of purine and pyrimidine ribosides to yield ribose and the bases. We were unable to detect NH activity of XopQ on standard substrates such as inosine, guanosine, thymidine, cytidine, and uridine. HopQ1 protein also does not appear to have $\mathrm{NH}$ activity ( $\mathrm{Li}$ et al. 2013a). We have found that $X$. oryzae pv. oryzae XopQ protein exhibits a very weak ribose hydrolase activity on the NH substrate analog, pNPR. Compared with wild-type XopQ, the XopQ ${ }^{D 116 \mathrm{~A}}$ and XopQ ${ }^{\text {Y279A }}$ mutant proteins exhibited reduced biochemical activity on pNPR. This is consistent with the idea that the phenotypes associated with the $\mathrm{XopQ}^{\mathrm{D} 116 \mathrm{~A}}$ and $\mathrm{XopQ}{ }^{\mathrm{Y} 279 \mathrm{~A}}$ mutant proteins are due to a deficiency in biochemical activity. However, we cannot rule out the possibility that, in addition to a reduction in biochemical activity, these mutations might have some other effect (such as interaction with 14-3-3 proteins) that is the prime reason for the phenotypes associated with the mutations.

We recognize that the ribose hydrolase activity on pNPR that we are describing for XopQ is very weak. This is likely to be due to the fact that pNPR is not the natural substrate for XopQ. We hypothesize that XopQ acts to hydrolyze some plant-specific ribose-harboring substrate. Recently, HopQ1 was found to act as a ribohydrolase on the cytokinin precursor IPRMP to elevate cytokinin levels and suppress FLS2 induced innate immunity in Arabidopsis (Hann et al. 2014). It is possible that XopQ protein may be having a similar biochemical activity. Alternatively, it could be acting on other substrates such as ADP-ribosylated plant proteins to release ribose.

Sinha and associates (2013) had reported that a xop $Q$ mutant of $X$. oryzae pv. oryzae exhibited a partial virulence deficiency on rice but was fully proficient at suppression of cell-walldegrading enzyme-induced host immune responses. This indicated that there was a redundancy for XopQ function with respect to its role in suppression of innate immunity but not with respect to its role in promoting virulence and suggested that, in addition to suppression of innate immunity, the XopQ protein has some other role in promoting virulence. The $\mathrm{XopQ}^{\mathrm{Y} 279 \mathrm{~A}}$ mutant is deficient in virulence but is proficient at suppressing PTI in rice and in Arabidopsis. This, again, is indicative that XopQ has an additional role in promoting virulence other than the ability to suppress innate immunity. What might be this additional function of XopQ in promoting virulence of $X$. oryzae pv. oryzae? One possibility is that, as suggested for HopQ1, the XopQ protein might be enhancing cytokinin levels in the host and this has some role in promoting virulence that is independent of the ability to suppress innate immunity.

In summary, the results presented here indicate that mutations which affect the biochemical activity of $X$. oryzae pv. oryzae XopQ also affect virulence on rice and induction of a HR in N. benthamiana. These results highlight the need for understanding the in vivo substrate for the biochemical activity of this protein. The mechanism by which $X$. oryzae pv. oryzae XopQ protein suppresses PTI in rice and Arabidopsis is not known, and it remains to be determined whether this occurs through interaction with host 14-3-3 proteins.

\section{MATERIALS AND METHODS}

\section{Bacterial strains, plasmids, primers, and culture media.}

$X$. oryzae pv. oryzae strains were grown at $28^{\circ} \mathrm{C}$ in peptone sucrose (PS) medium and E. coli strains were grown at $37^{\circ} \mathrm{C}$ in Luria Bertani medium, as described previously (Pandey and Sonti 2010). The bacterial strains and plasmids used are listed in Table 2. The primers used in this work are listed in Supplementary Table S2. The concentrations of antibiotics used in this study were as follows: rifampicin at $50 \mu \mathrm{g} / \mathrm{ml}$, ampicillin at $100 \mu \mathrm{g} / \mathrm{ml}$, spectinomycin at $50 \mu \mathrm{g} / \mathrm{ml}$, and kanamycin at 50 $\mu \mathrm{g} / \mathrm{ml}$ for $E$. coli and $15 \mu \mathrm{g} / \mathrm{ml}$ for $X$. oryzae pv. oryzae. In addition, cycloheximide $(80 \mu \mathrm{g} / \mathrm{ml})$ was added to the growth medium to control fungal contamination.

\section{Molecular tools and techniques.}

Plasmids were isolated by using the alkaline lysis method (Sambrook et al. 1989). High-fidelity Phusion polymerase (Thermo Scientific, Affibody AB, Solna, Sweden) was used for polymerase chain reaction (PCR) amplification. Restriction digestions and ligations were performed with enzymes from New England Biolabs (Beverly, MA, U.S.A.). PCR products and restriction enzyme-digested DNA fragments were purified using a QIAquick PCR purification kit and a QIAquick nucleotide removal kit (Qiagen, Hilden, Germany), respectively. Ligation with T4 DNA ligase, agarose gel electrophoresis, and transformation of $E$. coli were done as described by Sambrook and associates (1989).

\section{Sequencing and analysis.}

DNA sequencing was performed using an ABI Prism 3700 automated DNA sequencer (Perkin-Elmer, Foster City, CA, 
U.S.A.). The sequences were analyzed using the BLAST algorithm in the National Center for Biotechnology Information database (Altschul et al. 1990). Multiple sequence alignment was done using Clustal W (Thompson et al. 1994). ESPRIPT 3 (Gouet et al. 2003) was used to draw sequence alignment.
Generation and complementation

of the $X$. oryzae pv. oryzae $x o p Q$ mutant.

Gene $x o p Q$ of $X$. oryzae pv. oryzae was disrupted by homologous plasmid integration using suicide vector pK18mob (Schafer et al. 1994). To create a $x o p Q$ mutant, a 622-bp fragment internal to the $x o p Q$ gene was amplified by PCR using

Table 2. Plasmids and strains

\begin{tabular}{|c|c|c|}
\hline Strains & Relevant characteristics $^{\mathrm{a}}$ & Reference or source \\
\hline \multicolumn{3}{|l|}{ Escherichia coli strains } \\
\hline $\mathrm{DH} 5 \alpha$ & $\begin{array}{l}\lambda^{-} \text {f80dlacZDM15 D(lacZYA-argF) U169 recAl endA hsdR17 }\left(\mathrm{r}_{\mathrm{K}}{ }^{-} \mathrm{m}_{\mathrm{K}}{ }^{-}\right) \\
\text {supE44 thi-1 gyrA relA1 }\end{array}$ & Invitrogen \\
\hline BL21-AI & $\mathrm{F}^{-}$ompT hsd $S_{B}\left(\mathrm{r}_{\mathrm{B}}{ }^{-} \mathrm{m}_{\mathrm{B}}{ }^{-}\right)$gal dcmaraB::T7RNAP tetA & Invitrogen \\
\hline BL21-CodonPlus(DE3)-RIPL strain & 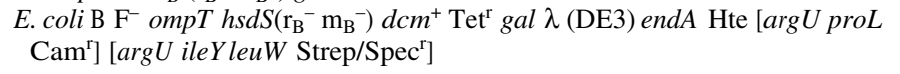 & Stratagene \\
\hline \multicolumn{3}{|l|}{ Xanthomonas oryzae pv. oryzae strains } \\
\hline BXO1 & Wild type; Indian isolate & Laboratory collection \\
\hline BXO43 & rif-2; derivative of $\mathrm{BXO} 1$ & Laboratory collection \\
\hline$x o p Q$ & xopQ::pK18mob rif-2; $\mathrm{Km}^{\mathrm{r}} ; \mathrm{XopQ}^{-}$, derivative of $\mathrm{BXO} 43$ & This work \\
\hline$x o p Q / \mathrm{pHM} 1$ & xopQ $Q: \mathrm{pK} 18 m o b / \mathrm{pHM} 1$ rif-2; $\mathrm{Km}^{\mathrm{r}}$; $\mathrm{XopQ}^{-}$, derivative of $x o p Q$ mutant & This work \\
\hline$x o p Q / \mathrm{pHM} 1:: x o p Q$ & $x o p Q:: \mathrm{pK} 18 m o b / \mathrm{pHM} 1-x o p Q$ rif-2; $\mathrm{Km}^{\mathrm{r}} ; \mathrm{XopQ}^{+}$, derivative of $x o p Q$ mutant & This work \\
\hline хорQ/pHM1::xop $Q^{D 116 A}$ & $\begin{array}{l}x o p Q:: \mathrm{pK} 18 m o b / \mathrm{pHM} 1-x o p Q^{D 116 A} \text { rif-2; } \mathrm{Km}^{\mathrm{r}} ; \mathrm{XopQ}^{\mathrm{D} 116 \mathrm{~A}} \text {, derivative of } x o p Q \\
\text { mutant }\end{array}$ & This work \\
\hline$x o p Q / \mathrm{pHM} 1:: x o p Q^{Y 279 A}$ & xop $Q:: \mathrm{pK} 18 m o b / \mathrm{pHM} 1-x o p Q^{Y 279 A}$ rif-2; $\mathrm{Km}^{\mathrm{r}} ; \mathrm{XopQ}^{\mathrm{Y} 279 \mathrm{~A}}$, derivative of $x o p Q$ & \\
\hline & mutant & This work \\
\hline$x o p Q / \mathrm{pHM} 1:: x o p Q^{D 116 A Y 279 A}$ & $\begin{array}{l}x o p Q:: \mathrm{pK} 18 m o b / \mathrm{pHM} 1-x o p Q^{D D 16 A} \text { Y279A } \\
\quad \text { of } x \text { if }-2 ; \mathrm{Km}^{\mathrm{r}} ; \mathrm{XopQ}^{\mathrm{D} 116 \mathrm{~A} \mathrm{Y} 279 \mathrm{~A}} \text {, derivative }\end{array}$ & This work \\
\hline $\mathrm{T} 3 \mathrm{~S}$ & hrpB6:: bla rif-2; $\mathrm{HR}^{-}, \mathrm{Ap}^{\mathrm{r}}$ derivative of $\mathrm{BXO} 43$ & Jha et al. 2007 \\
\hline xорN xоpQ xорX $x$ xopZ & xopN::pK18mob $\Delta x o p Q \Delta x o p X \Delta x o p Z ;$ rif-2; $\mathrm{Km}^{\mathrm{r}}$ & Sinha et al. 2013 \\
\hline xopN xopQ xopX $x$ xopZ/pHM1 & $\begin{array}{l}x o p N \text { xop } Q \text { xopX } x \text { opZ/pHM1; rif-2; } \mathrm{Km}^{\mathrm{r}}, \mathrm{Sp}^{\mathrm{r}} \text {; derivative of } x \text { opN } x \text { op } Q \text { xopX } \\
\quad \text { xopZ quadruple mutant }\end{array}$ & This work \\
\hline xорN xор $Q$ xорX хорZ/ pHM1::xop $Q$ & $\begin{array}{l}x o p N \text { xop } Q \text { xopX } x \text { opZ/pHM1::xop } Q ; \text { rif- } 2 ; \mathrm{Km}^{\mathrm{r}}, \mathrm{Sp}^{\mathrm{r}}, \mathrm{XopQ}^{+} \text {; derivative of } \\
\text { xopN } \text { xоp } Q \text { xopX xopZ quadruple mutant }\end{array}$ & This work \\
\hline xорN xорQ xорX $X$ xорZ/ pHM1::xop $Q^{D 116 A}$ & $\begin{array}{l}\text { xopN } x \text { opQ } Q \text { xopX } \times \text { opZ/pHM1::xop } Q^{D 116 A} ; \text { rif- } 2 ; \mathrm{Km}^{\mathrm{r}}, \mathrm{Sp}^{\mathrm{r}}, \mathrm{XopQ}^{\mathrm{D} 116 \mathrm{~A}} \\
\text { derivative of } x \text {; }\end{array}$ & This work \\
\hline xорN xоpQ $x$ хо X xopZ/ pHM1::xop $Q^{Y 279 A}$ & $\begin{array}{l}x o p N \text { xop } Q \text { xopX } x \text { opZ/pHM1::xop } Q^{Y 279 A} ; \text { rif- } 2 ; \mathrm{Km}^{\mathrm{r}}, \mathrm{Sp}^{\mathrm{r}}, \mathrm{XopQ}^{\mathrm{Y279A}} \text {; } \\
\text { derivative of } x \text { opN } x \text { op } Q \text { xopX } x \text { opZ quadruple mutant }\end{array}$ & This work \\
\hline xорN xор $Q$ хорX $X$ хорZ/ pHM1::xop $Q^{D 116 A Y 279 A}$ & $\begin{array}{l}x o p N \text { xopQ } x \text { opX XopZ/pHM1::xop } Q^{D 116 A}{ }^{279 A} ; \text { rif- } 2 ; \mathrm{Km}^{\mathrm{r}}, \mathrm{Sp}^{\mathrm{r}} \text {, } \\
\quad: \mathrm{XopQ}^{\text {D116A Y279A }} \text {; derivative of } x \text { opN } x \text { op } Q \text { xopX } x \text { opZ quadruple mutant }\end{array}$ & This work \\
\hline \multicolumn{3}{|l|}{ A. tumefaciens strains } \\
\hline EHA101 & $\begin{array}{l}\text { C58, pTiBo542; T-region::aph, } \mathrm{Km}^{\mathrm{r}} ; A 281 \text { derivative harboring } p E H A 101 \text {, } \\
\text { T-DNA replaced with } n p t \mathrm{II}, \text { super-virulent }\end{array}$ & Hood et al.1986 \\
\hline AGL1 & $\begin{array}{l}\text { AGL0 (C58 pTiBo542) recA::bla, T-region deleted } \mathrm{Mop}(+) \mathrm{Cb}(\mathrm{R})(\mathrm{AGL0} \text { is } \\
\text { an EHA101 with the T-region deleted) }\end{array}$ & Lazo et al.1991 \\
\hline AGL1/ pMDC7xop $Q$ & AGL1 carrying plasmid pMDC7xop $Q ; \mathrm{Sp}^{\mathrm{r}}$ & This work \\
\hline AGL1/ pMDC7 хор $Q^{Y 279 A}$ & AGL1 carrying plasmid pMDC $x \circ Q^{Y 279 A} ; \mathrm{Sp}^{\mathrm{r}}$ & This work \\
\hline \multicolumn{3}{|l|}{ Plasmids } \\
\hline pMDC7 & $17-\beta$-Estradiol inducible binary cloning vector; $\mathrm{Sp}^{\mathrm{r}}$ & $\begin{array}{l}\text { Curtis and } \\
\text { Grossniklauss } 2003\end{array}$ \\
\hline $\mathrm{pK} 18 m o b$ & pUC18 derivative; $\mathrm{Mob}^{+} \mathrm{Tra}^{-} \mathrm{Km}^{\mathrm{r}}$; does not replicate in $X$. oryzae pv. oryzae & Schafer et al. 1994 \\
\hline pENTR/D/TOPO & cloning vector; $\mathrm{Km}^{\mathrm{r}}$ & Invitrogen \\
\hline pENTRxopQ & $\begin{array}{l}\text { pENTR/D/TOPO with 1,392-bp fragment containing full length } x o p Q \text { gene } \\
\text { from BXO43 genomic DNA }\end{array}$ & This work \\
\hline pENTR $x o p Q^{Y 279 A}$ & pENTR/D/TOPO with 1,392-bp fragment containing full length $x o p Q^{Y 279 A}$ & This work \\
\hline pENTRxop $Q^{D 116 A}$ & $\begin{array}{l}\mathrm{pENTR/D/TOPO} \text { with 1,392-bp fragment containing full length } x o p Q^{D 116 A} \\
\text { gene from BXO43 genomic DNA }\end{array}$ & This work \\
\hline pMDC7xоp $Q$ & $\begin{array}{l}\text { pMDC7 with 1,392-bp fragment containing full length xop } Q \text { gene obtained } \\
\text { from recombination of pENTRxop } O \text { with pMDC7 }\end{array}$ & This work \\
\hline pMDC7 хор $Q^{Y 279 A}$ & $\begin{array}{l}\text { pMDC7 with } 1,392 \text {-bp fragment containing full length } x o p Q^{Y 279 A} \text { gene } \\
\text { obtained from recombination of pENTR xop } Q^{Y 279 A} \text { with pMDC7 }\end{array}$ & This work \\
\hline pMG1 & $\begin{array}{l}\text { pK18mob with 622-bp internal fragment of } x o p Q \text { gene of } X . \text { oryzae pv. oryzae } \\
\text { BXO43 cloned into SmaI site of pK18mob }\end{array}$ & This work \\
\hline pMG2 & $\begin{array}{l}\text { pHM1 with } 1,392 \text {-bp fragment containing full length } x o p Q \text { gene from genomic } \\
\text { DNA of wild type strain of } X \text {. oryzae pv. oryzae cloned into HindIII and }\end{array}$ & \\
\hline & $K p n I$ sites of $\mathrm{pHM} 1 ; \mathrm{Sp}^{\mathrm{r}}$ & This work \\
\hline pMG3 & $\begin{array}{l}\text { pHM1 with } 1,392-b p \text { fragment containing a full length } x o p Q^{D 116 A} \text { gene cloned } \\
\text { into HindIII and KpnI sites of pHM1; } \mathrm{Sp}^{\mathrm{r}}\end{array}$ & This work \\
\hline pMG4 & $\begin{array}{l}\text { pHM1 with 1,392-bp fragment containing a full length } x o p Q^{Y 279 A} \text { gene cloned } \\
\text { into HindIII and } K p n I \text { sites of } \mathrm{pHM} 1 ; \mathrm{Sp}^{\mathrm{r}}\end{array}$ & This work \\
\hline pMG5 & $\begin{array}{l}\text { pHM1 with 1,392-bp fragment containing full length } x o p Q^{D 116 A Y 279 A} \text { gene } \\
\text { cloned into HindIII and KpnI sites of } \mathrm{pHM} 1 ; \mathrm{Sp}^{\mathrm{r}}\end{array}$ & This work \\
\hline
\end{tabular}

${ }^{\mathrm{a}}$ The rif-2 mutation confers resistance to rifampicin; $\mathrm{Ap}^{\mathrm{r}}, \mathrm{Km}^{\mathrm{r}}$, $\mathrm{Cam}^{\mathrm{r}}$, Strep/Spec ${ }^{\mathrm{r}}$, Tet ${ }^{\mathrm{r}}$ and $\mathrm{Sp}^{\mathrm{r}}$ indicate resistance to ampicillin, $\mathrm{kanamycin}$, chloramphenicol, streptomycin/spectinomycin, and tetracycline respectively; and $\mathrm{HR}^{-}$indicates deficiency for hypersensitive response. 
genomic DNA of $X$. oryzae pv. oryzae $\mathrm{BXO} 43$ and the genespecific XopQF/XopQR primer pair and cloned into the pK18mob to generate the plasmid pMG1. The pMG1 was transferred into $X$. oryzae pv. oryzae by electroporation, and integration of the plasmid was selected by growth on peptone sucrose agar (PSA) containing rifampicin and kanamycin. Gene disruption was confirmed by PCR using flanking genespecific primers XopQUF/XopQUR (which bind in chromosomal regions that flank the insert sequence cloned in pK18 mob) in combination with vector-specific primers M13F and M13R and subsequent sequencing of the PCR products. In pMG1, the transcriptional orientation of the promoter of the lac $Z$ gene in $\mathrm{pK} 18 \mathrm{mob}$ and of the $x o p Q$ gene fragment were in the same direction, so that transcription from the lac $Z$ promoter would relieve any polar effect on transcription of downstream genes due to the mutation caused by pMG1 integration (Pandey and Sonti 2010; Schafer et al. 1994). One of the confirmed mutant clones (henceforth referred to as $x o p Q$ mutant) was chosen for further study. For $x o p Q$ mutant complementation, a 1,395-bp DNA fragment containing the entire XopQ coding region of the $x o p Q$ gene was amplified by PCR using the total DNA of $X$. oryzae pv. oryzae and primers pHM1XopQF/ pHM1XopQR. The amplified fragment was cloned as an HindIII-KpnI fragment into broad-host-range vector pHM1 (Innes et al. 1988) to create the recombinant plasmid pMG2. The pMG2 plasmid and pHM1 were individually introduced in to the $x o p Q$ mutant strain by electroporation to obtain $x o p Q^{-} /$ pHM1 (xop $Q^{-} / \mathrm{pHM} 1$, for vector control) and $x o p Q^{-} / \mathrm{pHM} 1:$ : xop $Q$ ( $x$ op $Q^{-} / \mathrm{pMG} 2$, for complementation), respectively.

Based on in silico modeling, we have identified two probable target sites (D116 and Y279) for mutagenesis which can disrupt ribose hydrolase activity of XopQ. For isolation of site-specific mutations, the $x o p Q$ gene was amplified using the $\mathrm{XopQF} /$ XopQR primer pair and cloned into pENTR/D-TOPO vector according to the manufacturer's instructions (Invitrogen, Carlsbad, CA, U.S.A.) and used as template for site-directed mutagenesis (SDM). The SDM for aspartic acid at 116 with alanine (D116A) and tyrosine at 279 with alanine (Y279A) was performed using the respective primer pairs $\left(\mathrm{XopQ}^{\mathrm{D} 116 \mathrm{~A}} \mathrm{~F} /\right.$ $\mathrm{XopQ}^{\mathrm{D} 116 \mathrm{~A}} \mathrm{R}$ for D116A and $\mathrm{XopQ}^{\mathrm{Y} 279 \mathrm{~A}} \mathrm{~F} / \mathrm{XopQ}^{\mathrm{Y} 279 \mathrm{~A}} \mathrm{R}$ for Y279A) and further confirmed through sequencing. The nucleotides as well as amino acid sequences for XopQ, $\mathrm{XopQ}^{\mathrm{D} 116 \mathrm{~A}}$, and XopQ ${ }^{\mathrm{Y} 279 \mathrm{~A}}$ were submitted in GenBank with accession numbers KJ809115, KJ809116, and KJ809117, respectively. The xopQ mutations having either a single mutation $\left(\operatorname{xop} Q^{D 116 A} ; \operatorname{xop} Q^{Y 279 A}\right)$ or a double mutation (xop $\left.Q^{D 116 A} Y 279 A\right)$ were subcloned into pHM1 vector to generate recombinant plasmids pMG3, pMG4, and pMG5, respectively. The pMG3, pMG4, and pMG5 plasmids were individually introduced into the $\operatorname{xop} Q$ mutant strain to obtain $x o p Q^{-} / \mathrm{pHM} 1:: x o p Q^{D 116 A}$ (xop $Q^{-/} \mathrm{pMG} 3$ ), $x o p Q^{-} /$ pHM1:: xop $Q^{Y 279 A}\left(x o p Q^{-} / \mathrm{pHM} 1:: \mathrm{pMG} 4\right)$, and double mutant xop $Q^{-} /$xop $Q^{D 116 A}$ Y279A $\left(x o p Q^{-} / \mathrm{pHM1}:\right.$ pMG5), respectively.

\section{Generation of $X$. oryzae pv. oryzae strains harboring pHM1, pHM1::xop $Q$, pHM1::xop $Q^{D 116 A}$, pHM1::xop $Q^{Y 279 A}$, and pHM1::xop $Q^{D 116 A}$ Y279A constructs in a quadruple mutant background.}

pHM1, pHM1::xop $Q$, pHM1::xop $Q^{D 116 A}$, pHM1::xop $Q^{Y 279 A,}$ and pHM1::xop $Q^{D 116 A Y 279 A}$ constructs were electroporated into electrocompetent cells of the $x o p N$ xop $Q$ xopX $x$ op $Z$ quadruple mutant of $X$. oryzae pv. oryzae (Sinha et al. 2013), followed by selection on media containing rifampicin, spectinomycin, and kanamycin.

\section{Assays for virulence and callose deposition in rice.}

$X$. oryzae pv. oryzae strains were grown to saturation and inoculated on 30- to 40-day-old glasshouse-grown rice plants of the highly susceptible rice 'Taichung Native' (TN-1). Inoculation was performed by dipping scissors into bacterial cultures and clipping the tips of rice leaves (Kauffman et al. 1973). Lesion lengths were measured at 10 days after inoculation and expressed as the mean lesion length with standard deviation. For callose assays, rice leaves were infiltrated with the $x o p N$ xop $Q$ xopX xopZ quadruple mutant of $X$. oryzae pv. oryzae harboring pHM1, pHM1::xop $Q$, pHM1::xop $Q^{D 116 A}$, pHM1::xop $Q^{Y 279 A}$, and pHM1::xop $Q^{D 116 A}$ Y279A constructs using a blunt-ended plastic syringe. After 20 to $24 \mathrm{~h}$, the leaves were cut and placed in absolute alcohol to remove chlorophyll followed by treatment with $70 \%$ ethanol at $65^{\circ} \mathrm{C}$, stained with $0.5 \%$ aniline blue for $4 \mathrm{~h}$, and observed under an Axioplan2 epifluorescence microscope (Carl Zeiss, Germany) using a blue filter (excitation wavelength of $365 \mathrm{~nm}$ ) and $\times 10$ objective (Hauck et al. 2003).

\section{Plant inoculation procedure} and in planta bacterial growth yield.

Plant inoculations and in planta bacterial growth analysis were performed on leaves of 45-day-old TN-1 rice plants. To monitor the growth of bacterial strains in planta, $X$. oryzae pv. oryzae wild type (BXO43), xop $Q$ mutant, mutant vector control (xop $\left.Q^{-} / \mathrm{pHM} 1\right)$, complement clone $\left(x \circ p Q^{-} / \mathrm{pHM} 1:\right.$ :xopQ $)$ single mutants (xop $Q^{-} / \mathrm{pHM} 1:$ : $x$ op $Q^{D 116 A} ;$ xop $Q^{-} / \mathrm{pHM} 1::$ $\left.x o p Q^{Y 279 A}\right)$, and double mutant (xop $Q^{-} / \mathrm{pHM} 1:$ :xop $\left.Q^{D I 16 A Y 279 A}\right)$ were grown to $\log$-phase in PS medium at $28^{\circ} \mathrm{C}$ for $24 \mathrm{~h}$ in the presence of appropriate antibiotics to obtain preinoculum. For each strain, $20 \mathrm{ml}$ of PS medium was inoculated with $0.1 \%$ preinoculum. Cultures were grown for $24 \mathrm{~h}$. Cultures were pelleted down and diluted to an optical density at $600 \mathrm{~nm}\left(\mathrm{OD}_{600}\right)$ of 1.0 and clip inoculated on rice leaves. Leaf samples were collected immediately after inoculation (day 0) and 6 days after inoculation. In order to estimate bacterial counts in the plant, $1.5-\mathrm{cm}^{2}$ leaf discs were cut down and ground in $0.2 \%$ Silwet L-77. Subsequent serial dilutions and plating were performed by using PSA plates with rifampicin, cephalexin, and cyclohexamide antibiotics to suppress growth of contaminating bacteria and fungi. The bacterial count was expressed as the $\log$ value of CFU/1.5 $\mathrm{cm}^{2}$ (Fig. 3).

\section{$X$. oryzae pv. oryzae infiltration and $\mathrm{HR}$ in $N$. benthamiana.}

For HR assays, $X$. oryzae pv. oryzae wild type (BXO43), $x o p Q$ mutant, mutant vector control ( $\left.x o p Q^{-} / \mathrm{pHM} 1\right)$, complement clone (xop $\left.Q^{-} / \mathrm{pHM} 1:: x o p Q\right)$ single mutants (xop $Q^{-}$ /pHM1::xop $Q^{D 116 A}$ and $\left.x o p Q^{-} / \mathrm{pHM} 1:: x o p Q^{Y 279 A}\right)$, and double mutant (xop $Q^{-} / \mathrm{pHM} 1:$ xop $Q^{\text {D116A Y279A }}$ ) were grown to logphase in PS medium at $28^{\circ} \mathrm{C}$ in the presence of appropriate antibiotics. The cultures were pelleted down by centrifugation and resuspended in Milli-Q (MQ) to a final $\mathrm{OD}_{600}$ of 0.5 . N. benthamiana plants were grown in a growth chamber with 16 $\mathrm{h}$ of lights (30 to $40 \mu \mathrm{mol} \mathrm{s} \mathrm{m}^{-2}$ ) (Li et al. 2013a). Further, the cultures were directly infiltrated into the abaxial surfaces of leaves from 6- to 7-week-old plants, using a needleless 1-ml syringe. HR symptoms at the area of inoculation were observed $60 \mathrm{~h}$ after infiltration by trypan blue staining. For this purpose, leaves were stained with lactophenol-trypan blue $(10 \mathrm{ml}$ of lactic acid, $10 \mathrm{ml}$ of glycerol, $10 \mathrm{ml}$ of distilled water, $10 \mathrm{~g}$ of phenol, and $10 \mathrm{mg}$ of trypan blue) (Koch and Slusarenko 1990). Leaf samples were boiled for approximately $1 \mathrm{~min}$ in the lactophenol-trypan blue solution and then decolorized in chloral hydrate $(2.5 \mathrm{~g}$ of chloral hydrate dissolved in $1 \mathrm{ml}$ of distilled water) for $5 \mathrm{~min}$.

\section{Measurement of electrolyte leakage in $N$. benthamiana.}

For the electrolyte leakage assays, two leaf discs $(11 \mathrm{~mm}$ in diameter; one disc per leaf) were collected from the infiltrated zone in leaves infiltrated with one of the following: $X$. oryzae 
pv. oryzae wild type (BXO43), xop $Q$ mutant, mutant vector control (xop $\left.Q^{-} / \mathrm{pHM} 1\right)$, complement clone $\left(x o p Q^{-}\right.$ $/ \mathrm{pHM} 1:: x o p Q)$, single mutants (xop $Q^{-} / \mathrm{pHM} 1:: x o p Q^{D 116 A}$ and $x o p Q^{-} / \mathrm{pHM} 1:$ xop $Q^{Y 279 A}$ ), and the double mutant (xop $Q^{-}$ /pHM1::xop $Q^{\text {D116A Y279A }}$ ). The leaf discs were incubated in 10 $\mathrm{ml}$ of nonionic double-distilled water for $2 \mathrm{~h}$ at $25^{\circ} \mathrm{C}$ with shaking at $160 \mathrm{rpm}$. Leaf discs were then removed and ion conductivity of the solution was measured using a Thermo Scientific Orion conductivity meter (Orion star A325; Thermo Scientific, Beverly, MA, U.S.A.).

\section{Chemiluminescent Western blotting.}

Twelve-day-old greenhouse-grown rice seedlings of the susceptible rice $\mathrm{TN}-1$ were clip inoculated with sterile scissors dipped in cultures of the following $X$. oryzae pv. oryzae strains: wild type (BXO43), xop $Q$ mutant, $x$ op $Q^{-} / \mathrm{pHM} 1:: x \circ p Q$ (complemented strain), xop $Q^{-} / \mathrm{pHM} 1:$ xop $Q^{Y 279 A}, \quad$ xop $Q^{-}$ /pHM1::xop $Q^{D 116 A}$, and $x o p Q^{-} / \mathrm{pHM} 1: \operatorname{xop} Q^{D 116 A}$ Y279A. Four days after inoculation, $2-\mathrm{cm}$ leaf pieces were cut from the clipped end of the leaf and ground in liquid $\mathrm{N}_{2}$ followed by homogenization in lysis buffer $(50 \mathrm{mM}$ Tris- $\mathrm{HCl}$ [pH 8.0], 250 $\mathrm{mM} \mathrm{NaCl}, 10 \mathrm{mM} \beta$-mercaptoethanol, $1 \%$ Triton X100, $1 \mathrm{mM}$ PMSF, plant protease inhibitor cocktail for plant cell, and tissue extracts [P9599; Sigma-Aldrich]). Total protein supernatants were isolated after centrifugation at $14,000 \mathrm{rpm}$ for 15 min at $4{ }^{\circ} \mathrm{C}$ to remove cellular debris (Nomura et al. 2006). Equal amounts of isolated protein supernatants were further used for sodium dodecyl sulfate polyacrylamide gel electrophoresis (SDS-PAGE) or Western blotting. XopQ wild-type and mutant proteins were detected by Western blot analysis using anti:XopQ antibodies raised in rabbit. HRP conjugated to antirabbit immunoglobulin $\mathrm{G}$ (ab98440) and chemiluminescent reagent Luminata Forte (WBLUF0100) were used for detecting protein signals using the Vilber Lourmat chemiluminescence imaging system with Chemi-capt 5000 software (version 12.8; Vilber Lourmat, Eberhardzell, Germany).

\section{Electroporation of recombinant construct into Agrobacterium AGL1.}

Recombinant plasmid (100 ng) of pMDC7xop $Q$ and pMDC7 xop $Q^{Y 279 A}$ constructs was mixed with $100 \mu$ of electrocompetent cells of AGL1 separately and kept in ice for $20 \mathrm{~min}$. After electroporation, $1 \mathrm{ml}$ of tryptone yeast extract and mannitol (TYM) medium was added instantly and the Agrobacterium cells were incubated at $28^{\circ} \mathrm{C}$ for $2 \mathrm{~h}$. The transformation mixture was plated on TYM agar plates containing rifampicin $(50 \mu \mathrm{g} / \mathrm{ml})$, cephalexin $(20 \mu \mathrm{g} / \mathrm{ml})$, cycloheximide $(75 \mu \mathrm{g} / \mathrm{ml})$, and spectinomycin $(50 \mu \mathrm{g} / \mathrm{ml})$. Plates were incubated at $28^{\circ} \mathrm{C}$ for 36 to $48 \mathrm{~h}$. The transformed colonies were picked for confirmation studies. Colony PCR was performed with gene-specific primers and glycerol stocks were prepared of the confirmed clones.

\section{Generation of a Gateway vector for N-terminally \\ His-tagged wild-type XopQ protein and mutants.}

The pDEST17xop $Q$ vector was constructed by amplifying the full-length coding region of the XopQ gene with forward and reverse primers. This amplicon was first cloned into the $\mathrm{pENTR/D-TOPO} \mathrm{vector} \mathrm{(Invitrogen)} \mathrm{and,} \mathrm{further,} \mathrm{the} \mathrm{gene}$ was subcloned into pDEST17 vector by LR reactions (Invitrogen) using the manufacturer's instructions. Similarly, the identified putative catalytic residues D116 and Y279 were mutated into alanine using pENTR XopQ as template (described below in the section on structural modeling and site-directed mutagenesis). pENTR xop $Q^{D 116 A}$ and pENTR xop $Q^{Y 279 A}$ were subcloned into $\mathrm{pDEST} 17$ vector.

The pDEST17xop $Q$, pDEST17 xop $Q^{D 116 A}$, and pDEST17 xop $Q^{Y 279 A}$ constructs were then transformed into BL21 codon plus host cells for overexpression. The overexpressed XopQ and XopQ mutant proteins are detected in the soluble fraction. The overexpressed proteins were purified and eluted using Talon metal affinity resin followed by gel filtration using a Superdex 200 column. SDS-PAGE analysis followed by Coomassie staining of the purified proteins is shown in Supplementary Figure S3.

\section{Enzyme assay.}

Ribose hydrolase activity for XopQ and its mutant versions was performed with purified proteins by using the chromogenic substrate analogue pNPR, which gives rise to p-nitrophenol (a colored product) with maximum absorbance at $405 \mathrm{~nm}$. The assay mixture contained purified $0.84 \mu \mathrm{M}$ enzyme, $50 \mathrm{mM}$ HEPES ( $\mathrm{pH} 8.0$ ), and $50 \mu \mathrm{M}$ substrate in a total reaction volume of $200 \mu \mathrm{l}$. The change in absorbance was determined at $405 \mathrm{~nm}$. To test the NH activity of XopQ on nucleosides such as inosine, guanosine, thymidine, cytidine, and uridine, we used the protocol as described by Parkin (1996).

\section{Structural modeling and site directed mutagenesis.}

The sequence similarity of XopQ (Xoo_4208) with the known structures present in the PDB is low. Hence, the structural model of XopQ was prepared using the online server Swiss-model (Arnold et al. 2006), using automated mode. The search used the already known crystal structure of inosineuridine-preferring $\mathrm{NH}$ from B. anthracis (PDB number: 2C40) and generated a model for XopQ having a sequence length from 109 to 430 amino acids. In the absence of any structural and functional studies corresponding to the crystal structure of the inosine-uridine-preferring $\mathrm{NH}$ from $B$. anthracis, another crystal structure of purine NH from $C$. fasciculata (PDB number: 2MAS) was used to further analyze the predicted structural model of XopQ. The crystal structure of purine NH is cocomplexed with an enzyme inhibitor, 2-(4-amino-phenyl)-5hydroxymethyl-pyrrolidine-3, 4-diol (PIR), in the active site.

Based on modeling of the inhibitor PIR in the predicted active site in the XopQ model, the putative catalytic residues D116 and Y279 were selected for further study. These two identified target sites for mutagenesis were cloned into the pENTR plasmid vector and mutated using the synthesis of respective oligos with the desired point mutation (at 116 and 279 amino acid positions, the codon was converted to code for alanine). The single-stranded primer was then extended using a Phusion polymerase, which copied the rest of the gene. The gene thus copied contained the mutated site, which was confirmed by sequencing.

\section{Generation of pMDC7xop $Q$ and pMDC7xop $Q^{Y 279 A}$ transgenic Arabidopsis plants.}

The Agrobacterium tumefaciens strain (AGL1) is hypervirulent but attenuated for tumor induction. It has a helper plasmid, pTiBo542 (from which the T-DNA region sequences have been precisely deleted), which promotes efficient T-DNA transfer from co-resident binary plasmids. Agrobacterium-mediated in planta floral-dip transformation was carried out using the method of Clough and Bent (1998), with minor modifications. Agrobacterium cell cultures harboring the construct in the binary vector (pMDC7xopQ and pMDC7 $x \circ p Q^{Y 279 A}$ ) were grown in TYM broth with spectinomycin $(50 \mu \mathrm{g} / \mathrm{ml})$ at $28^{\circ} \mathrm{C}$ in a rotary shaker till midlog phase. The cells were then pelleted down and resuspended in a solution containing 5\% sucrose and $0.05 \%$ Silwet L-77. The $\mathrm{T}_{1}$ seed were plated on Murashige Skoog medium containing hygromycin $(25 \mu \mathrm{g} / \mathrm{ml})$ and cefotaxime $(100 \mu \mathrm{g} / \mathrm{ml})$ supplemented with $2 \%$ sucrose. Approximately $30 \mathrm{~T}_{1}$ seedlings (that were green and tall, as opposed to the sensitive plants, which were short) were obtained. The 
green and tall seedlings were later transplanted to pots for growth until maturity. The seed of these plants were collected and germinated on hygromycin media to obtain the $\mathrm{T}_{2}$ generation. The hygromycin-resistant seedlings were transferred to pots for seed collection and for further analysis.

\section{Callose assay in Arabidopsis roots.}

Callose deposition assays in Arabidopsis roots were performed with slight modifications from the protocol described by Millet and associates (2010). Twelve-day-old seedlings of transgenic Arabidopsis plants (pMDC7xopQ and pMDC7 xop $Q^{Y 279 A}$ ) and wild-type Arabidopsis thaliana Col-0 roots were kept in one of the following: buffer or purified LipA (approximately $100 \mu \mathrm{g} / \mathrm{ml})$ with and without $17-\beta$-estradiol $(20 \mu \mathrm{M}$ final concentration). Plants were left in a growth chamber and roots were harvested after $12 \mathrm{~h}$. Harvested roots were fixed in ethanol/acetic acid (3:1) solution for $4 \mathrm{~h}$ and changed regularly to ensure complete clearing of the roots. Seedlings were further rehydrated in $70 \%$ ethanol for $2 \mathrm{~h}, 50 \%$ ethanol for $2 \mathrm{~h}$, and MQ water for $2 \mathrm{~h}$ and were stained with $0.01 \%$ aniline blue for several hours. Callose deposits were monitored with an Axioplan2 epifluorescence microscope (Carl Zeiss) using a blue filter (excitation wavelength of $365 \mathrm{~nm}$ ) and $\times 10$ objective (Hauck et al. 2003).

\section{ACKNOWLEDGMENTS}

We thank N. H. Chua for providing the plasmid pMDC7 and A. Pandey and M. G. Anil for critical reading of the manuscript. This work was supported by grants to R. V. Sonti from the Plant-Microbe and Soil Interactions project of the Council of Scientific and Industrial Research and the Department of Biotechnology (DBT), Government of India. M. K. Gupta acknowledges a DBT postdoctoral fellowship.

\section{LITERATURE CITED}

Akimoto-Tomiyama, C., Furutani, A., Tsuge, S., Washington, E. J., Nishizawa, Y., Minami, E., and Ochiai, H. 2012. XopR, a type III effector secreted by Xanthomonas oryzae pv. oryzae, suppresses microbeassociated molecular pattern-triggered immunity in Arabidopsis thaliana. Mol. Plant-Microbe Interact. 25:505-514.

Alfano, J. R., and Collmer, A. 1997. The type III (Hrp) secretion pathway of plant pathogenic bacteria: Trafficking hairpins, Avr proteins, and death. J. Bacteriol. 179:5655-5662.

Altschul, S., Gish, W., Miller, W., Myers, E., and Lipman, D. 1990. Basic local alignment search tool. J. Mol. Biol. 215:403-410.

Arnold, K., Bordoli, L., Kopp, J., and Schwede, T. 2006. The SWISSMODEL Workspace: A web-based environment for protein structure homology modelling. Bioinformatics 22:195-201.

Cleland, W. W. 1979. Statistical analysis of enzyme kinetic data. Methods Enzymol. 63:103-138.

Clough, S. J., and Bent, A. F. 1998. Floral dip: A simplified method for Agrobacterium mediated transformation of Arabidopsis thaliana. Plant J. 16:735-743.

Curtis, M. D., and Grossniklaus, U. 2003. A gateway cloning vector set for high-throughput functional analysis of genes in planta. Plant Physiol. 133:462-469

Degano, M., Gopaul, D. N., Scapin, G., Schramm, V. L., and Sacchettini, J. C. 1996. Three-dimensional structure of the inosine-uridine nucleoside N-ribohydrolase from Crithidia fasciculata. Biochemistry 35:5971-5981.

Degano, M., Almo S. C., Sacchettini J. C., and Schramm, V. L. 1998. Trypanosomal nucleoside hydrolase. A novel mechanism from the structure with a transition-state inhibitor. Biochemistry 37:6277-6285.

Furutani, A., Takaoka, M., Sanada, H., Noguchi, Y., Oku, T., Tsuno, K., Ochiai, H., and Tsuge, S. 2009. Identification of novel type III secretion effectors in Xanthomonas oryzae pv. oryzae. Mol. Plant-Microbe Interact. 22:96-106.

Giabbai, B, and Degano, M. 2004. Crystal structure to $1.7 \AA$ of the Escherichia coli pyrimidine nucleoside hydrolase YeiK, a novel candidate for cancer gene therapy. Structure 12:739-749.

Giska, F., Lichocka, M., Piechocki, M., Dadlez, M., Schmelzer, E., Hennig, J., and Krzymowska, M. 2013. Phosphorylation of HopQ1, a type III effector from Pseudomonas syringae, creates a binding site for host 14-
3-3 proteins. Plant Physiol. 161:2049-2061.

Gouet, P., Robert, X., and Courcelle, E. 2003. ESPript/ENDscript: Extracting and rendering sequence and $3 \mathrm{D}$ information from atomic structures of proteins. Nucleic Acids Res. 31:3320-3323.

Hann, D. R., Domínguez-Ferreras, A., Motyka, V., Dobrev, P. I., Schornack, S., Jehle, A., Felix, G., Chinchilla, D., Rathjen, J. P., and Boller, T. 2014. The Pseudomonas type III effector HopQ1 activates cytokinin signaling and interferes with plant innate immunity. New Phytol. 201:585-598.

Hauck, P., Thilmony, R., and He, S. Y. 2003. A Pseudomonas syringae type III effector suppresses cell wall-based extracellular defense in susceptible Arabidopsis plants. Proc. Natl. Acad. Sci. U.S.A. 100:8577-8582.

Hood, E. E., Helmer, G. L., Fraley, R. T., and Chilton, M.-D. 1986. The hypervirulence of Agrobacterium tumefaciens A281 is encoded in a region of pTiBo542 outside of T-DNA. J Bacteriol. 168:1291-1301.

Innes, R. W., Hirose, M. A., and Kuempel, P. L. 1988. Induction of nitrogen-fixing nodules on clover requires only 32 kilobase pairs of DNA from the Rhizobium trifolii symbiosis plasmid. J. Bacteriol. 170:37933802

Jha, G., Rajeshwari, R., and Sonti, R. V. 2007. Functional interplay between two Xanthomonas oryzae pv. oryzae secretion systems in modulating virulence on rice. Mol. Plant-Microbe Interact. 20:31-40.

Jiang, B. L., He, Y. Q., Cen, W. J., Wei, H. Y., Jiang, G. F., Jiang, W., Hang, X. H., Feng, J. X., Lu, G. T., Tang, D. J., and Tang, J. L. 2008. The type III secretion effector XopXccN of Xanthomonas campestris pv. campestris is required for full virulence. Res. Microbiol. 159:216220.

Kauffman, H. E., Reddy, A. P. K., Hsieh, S. P. Y., and Merca, S. D. 1973. An improved technique for evaluation of resistance of rice varieties to Xanthomonas oryzae. Plant Dis. Rep. 57:537-541.

Kim, J. G., Li, X., Roden, J. A., Taylor, K. W., Aakre, C. D., Su, B., Lalonde, S., Kirik, A., Chen, Y., Baranage, G., McLane, H., Martin, G. B., and Mudgett, M. B. 2009. Xanthomonas T3S effector XopN suppresses PAMP-triggered immunity and interacts with a tomato atypical receptor-like kinase and TFT1. Plant Cell 21:1305-1323.

Koch, E., and Slusarenko, A. 1990. Arabidopsis is susceptible to infection by a downy mildew fungus. Plant Cell 2:437-445.

Lazo, G. R., Stein, P. A., Ludwig, R. A. 1991. A DNA transformationcompetent Arabidopsis genomic library in Agrobacterium. Biotechnology 9:963-967.

Lee, B. M., Park, Y. J, Park, D. S., Kang, H. W., Kim, J. G., Song, E. S., Park, I. C., Yoon, U. H., Hahn, J. H., Koo, B. S., Lee, G. B., Kim, H., Park, H. S., Yoon, K. O., Kim, J. H., Jung, C. H., Koh, N. H., Seo, J. S., and Go, S. J. 2005. The genome sequence of Xanthomonas oryzae pv. oryzae KACC10331, the bacterial blight pathogen of rice. Nucleic Acids Res. 33:577-586.

Li, W., Chiang, Y.-H., and Coaker, G. 2013a. The HopQ1 effector's nucleoside hydrolase-like domain is required for bacterial virulence in Arabidopsis and tomato, but not host recognition in tobacco. PLoS One 8:e59684. Published online.

Li, W, Yadeta, K. A., Elmore, J. M., and Coaker, G. 2013b. The Pseudomonas syringae effector HopQ1 promotes bacterial virulence and interacts with tomato 14-3-3 proteins in a phosphorylation-dependent manner. Plant Physiol. 161:2062-2074.

Metz, M., Dahlbeck, D., Morales, C. Q., Al Sady, B., Clark, E. T., and Staskawicz, B. J. 2005. The conserved Xanthomonas campestris pv. vesicatoria effector protein $\mathrm{XopX}$ is a virulence factor and suppresses host defense in Nicotiana benthamiana. Plant J. 41:801-814.

Millet, Y. A., Danna, C. H., Clay, N. K., Songnuan, W., Simon, M. D., Werck-Reichhart, D., and Ausubel, F. M. 2010. Innate immune responses activated in Arabidopsis roots by microbe-associated molecular patterns. Plant Cell 22:973-990.

Nicaise, V., Roux, M., and Zipfel, C. 2009. Recent advances in PAMPtriggered immunity against bacteria: Pattern recognition receptors watch over and raise the alarm. Plant Physiol. 150:1638-1647.

Nomura, K., Debroy, S., Lee, Y. H., Pumplin, N., Jones, J., and He, S.Y. 2006. A bacterial virulence protein suppresses host innate immunity to cause plant disease. Science. 313:220-223.

Ochiai, H., Inoue, Y., Takeya, M., Sasaki, A., and Kaku, H. 2005. Genome sequence of Xanthomonas oryzae pv. oryzae suggests contribution of large numbers of effector genes and insertion sequences to its race diversity. JARQ 39:275-287.

Pandey, A., and Sonti, R. V. 2010. Role of the FeoB protein and siderophore in promoting virulence of Xanthomonas oryzae pv. oryzae on rice. J. Bacteriol. 192:3187-3203.

Parkin, D. W. 1996. Purine-specific Nucleoside N-Ribohydrolase from Trypanosoma brucei brucei. J. Biol. Chem. 271:21713-21719.

Salzberg, S. L., Sommer, D. D., Schatz, M. C., Phillippy, A. M., Rabinowicz, P. D., Tsuge, S., Furutani, A., Ochiai, H., Delcher, A. L., Kelley, D., Madupu, R., Puiu, D., Radune, D., Shumway, M., Trapnell, C., Aparna, 
G., Jha, G., Pandey, A., Patil, P. B., Ishihara, H., Meyer, D. F., Szurek, B., Verdier, V., Koebnik, R., Dow, J. M., Ryan, R. P., Hirata, H., Tsuyumu, S., Won Lee, S., Seo, Y. S., Sriariyanum, M., Ronald, P. C., Sonti, R. V., Van Sluys, M. A., Leach, J. E., White, F. F., and Bogdanove, A. J. 2008. Genome sequence and rapid evolution of the rice pathogen Xanthomonas oryzae pv. oryzae PXO99A. BMC Genomics 9:204.

Sambrook, J., Fritsch, E., and Maniatis, T. 1989. Molecular Cloning: A Laboratory Manual. Cold Spring Harbor Laboratory Press, Cold Spring Harbor, NY, U.S.A.

Schäfer, A., Tauch, A., Jäger, W., Kalinowski, J., Thierbach, G., and Pühler, A. 1994. Small mobilizable multi-purpose cloning vectors derived from the Escherichia coli plasmids pK18 and pK19: Selection of defined deletions in the chromosome of Corynebacterium glutamicum. Gene 145:69-73.

Sinha, D., Gupta, M. K., Patel, H. K., Ranjan, A., and Sonti, R. V. 2013. Cell-wall-degrading enzyme-induced rice innate immune responses are suppressed by the Type 3 secretion system effectors XopN, XopQ, XopX and XopZ of Xanthomonas oryzae pv. oryzae. PLoS One 8:e75867. Published online.

Song, C., and Yang, B. 2010. Mutagenesis of 18 Type III effectors reveals virulence function of XopZPXO99 in Xanthomonas oryzae pv. oryzae. Mol. Plant-Microbe Interact. 23:893-902.

Teper, D., Salomon, D., Sunitha, S., Kim, J.-G., Mudgett, M. B., and Sessa, G. 2014. Xanthomonas euvesicatoria type III effector XopQ interacts with tomato and pepper 14-3-3 isoforms to suppress effectortriggered immunity. Plant J. 77:297-309.
Thompson, J. D., Higgins, D. G., and Gibson, T. J. 1994. CLUSTAL W: Improving the sensitivity of progressive multiple sequence alignment through sequence weighting, position specific gap penalties and weight matrix choice. Nucleic Acids Res. 22:4673-4680.

Versées, W and Steyaert, J. 2003. Catalysis by nucleoside hydrolases. Curr. Opin. Struct. Biol. 13:731-738.

Wei, C.-F., Kvitko, B. H., Shimizu, R., Crabill, E., Alfano, J. R., Lin, N.C., Martin, G. B., Huang, H.-C., and Collmer, A. 2007. A Pseudomonas syringae pv. tomato DC3000 mutant lacking the type III effector HopQ1-1 is able to cause disease in the model plant Nicotiana benthamiana. Plant J. 51:32-46.

Yu, S., Hwang, I., and Rhee, S. 2013. Crystal structure of the effector protein XOO4466 from Xanthomonas oryzae. J. Struct. Biol. 184:361-366.

Zhao, S., Mo, W. L., Wu, F., Tang, W., Tang, J. L., Szurek, B., Verdier, V., Koebnik, R., and Feng, J. X. 2013. Identification of non-TAL effectors in Xanthomonas oryzae pv. oryzae Chinese strain 13751 and analysis of their role in the bacterial virulence. World J. Microbiol. Biotechnol. 29:733-744.

Zhu, W., Magbanua, M. M., and White, F. F. 2000. Identification of two novel hrp associated genes in the hrp gene cluster of Xanthomonas oryzae pv. oryzae. J. Bacteriol. 182:1844-1853.

\section{AUTHOR-RECOMMENDED INTERNET RESOURCE}

ExPASy server's Swiss-model: www.swissmodel.expasy.org 